ARTICLE

https://doi.org/10.1038/s41467-018-07911-6

\title{
RORyt inhibition selectively targets IL-17 producing iNKT and $\gamma \delta$-T cells enriched in Spondyloarthritis patients
}

Koen Venken ${ }^{1,2}$, Peggy Jacques ${ }^{1,2}$, Céline Mortier ${ }^{1,2}$, Mark E. Labadia ${ }^{3}$, Tine Decruy ${ }^{1,2}$, Julie Coudenys ${ }^{1,2}$, Kathleen Hoyt ${ }^{3}$, Anita L. Wayne ${ }^{3}$, Robert Hughes ${ }^{3}$, Michael Turner ${ }^{3}$, Sofie Van Gassen ${ }^{2,4}$, Liesbet Martens (1) 2,4, Dustin Smith ${ }^{3}$, Christian Harcken ${ }^{3}$, Joseph Wahle ${ }^{3}$, Chao-Ting Wang ${ }^{3}$, Eveline Verheugen ${ }^{1,2}$, Nadia Schryvers ${ }^{1,2}$, Gaëlle Varkas ${ }^{1,2}$, Heleen Cypers ${ }^{1,2}$, Ruth Wittoek ${ }^{1,2}$, Yves Piette ${ }^{1,2,5}$, Lieve Gyselbrecht ${ }^{6}$, Serge Van Calenbergh (10 7, Filip Van den Bosch ${ }^{1,2}$, Yvan Saeys ${ }^{2,4}$, Gerald Nabozny ${ }^{3}$ \& Dirk Elewaut ${ }^{1,2}$

Dysregulated IL-23/IL-17 responses have been linked to psoriatic arthritis and other forms of spondyloarthritides (SpA). RORyt, the key Thelper17 (Th17) cell transcriptional regulator, is also expressed by subsets of innate-like T cells, including invariant natural killer T (iNKT) and $\gamma \delta$-T cells, but their contribution to SpA is still unclear. Here we describe the presence of particular ROR $\gamma \mathrm{t}^{+}{ }^{\mathrm{T}}$-bet ${ }^{\mathrm{loPLZF}}{ }^{-}$iNKT and $\gamma \delta$-hi $\mathrm{T}$ cell subsets in healthy peripheral blood. ROR $\gamma \mathrm{t}^{+}$iNKT and $\gamma \delta$-hi T cells show IL-23 mediated Th17-like immune responses and were clearly enriched within inflamed joints of SpA patients where they act as major IL-17 secretors. SpA derived iNKT and $\gamma \delta$-T cells showed unique and Th17-skewed phenotype and gene expression profiles. Strikingly, ROR $\gamma$ t inhibition blocked $\gamma \delta 17$ and iNKT17 cell function while selectively sparing IL-22+ subsets. Overall, our findings highlight a unique diversity of human ROR $\gamma \mathrm{t}^{+} \mathrm{T}$ cells and underscore the potential of ROR $\gamma$ t antagonism to modulate aberrant type 17 responses.

\footnotetext{
${ }^{1}$ Department of Rheumatology, Faculty of Medicine and Health Sciences, Laboratory for Molecular Immunology and Inflammation, Ghent University, C. Heymanslaan 10, 9000 Ghent, Belgium. ${ }^{2}$ VIB Inflammation Research Center, Ghent University, Technologiepark 71, 9052 Ghent, Belgium. ${ }^{3}$ Research and Development Boehringer-Ingelheim, 175 Briar Ridge Road, Ridgefield, CT 06877, USA. ${ }^{4}$ Department of Applied Mathematics, Computer Science and Statistics, Ghent University, Technologiepark 71, 9052 Ghent, Belgium. ${ }^{5}$ AZ SintJan, Ruddershove 10, 8000 Brugge, Belgium. ${ }^{6}$ ASZ Aalst, Merestraat 80, 9300 Aalst, Belgium. ${ }^{7}$ Laboratory for Medicinal Chemistry, Faculty of Pharmaceutical Sciences, Ghent University, Ottergemsesteenweg 460, 9052 Gent, Belgium. Correspondence and requests for materials should be addressed to K.V. (email: koen.venken@ugent.be) or to D.E. (email: dirk.elewaut@ugent.be)
} 
S pondyloarthritides $(\mathrm{SpA})$ refers to a cluster of inflammatory rheumatic diseases including ankylosing spondylitis (AS) and psoriatic arthritis (PsA), affecting nearly $1-2 \%$ of the Western population. Typical disease manifestations consist of inflammation of sacroiliac joints, as well as of the spine. Peripheral joints can also be affected (peripheral arthritis), as well as insertion of tendons to bone (enthesitis) ${ }^{1}$. Importantly, the disease also causes inflammation and tissue damage beyond the musculoskeletal system with the most typically affected organs being the eye (anterior uveitis), skin (psoriasis), and gut (inflammatory bowel disease-IBD). This association is further substantiated by a significant overlap in the underlying genetic predisposition for these disorders ${ }^{2}$. While the role for TNFa as therapeutic target in SpA has been documented widely, an emerging role for the IL-23/IL-17 inflammatory axis in this disease has arisen as evidenced by the marked efficacy of IL-17a inhibition (AS and PsA), as well as IL-12 and/or IL-23 blockade (PsA $)^{3-5}$. Curiously, IL-17 inhibition failed to demonstrate efficacy in patients with rheumatoid arthritis (RA) and IBD ${ }^{6,7}$. IL-23 and IL-17 show divergent roles on epithelial barrier integrity in experimental models of gut inflammation ${ }^{8,9}$, which could explain the dichotomy of clinical efficacy of anti-IL-17 vs. IL-12/IL-23 inhibition in IBD patients, even though both are efficacious on joint symptoms in SpA. IL-22, the other IL-23 signature cytokine, also plays a key role in the maintenance of mucosal homeostasis by promotion of antimicrobial immunity, inflammation, and tissue repair at barrier surfaces ${ }^{10}$. However, it is not completely understood how differential expression of IL-17 and IL-22 is regulated and potentially disturbed at the cellular level in $\mathrm{SpA}^{3}$.

IL-23 is necessary for the terminal differentiation and inflammatory functions associated with $\mathrm{T}$ helper-17 cells (Th17), characterized by the expression of the key transcription factor retinoic acid receptor-related orphan receptor-yt (ROR $y$ t; which is encoded by $R O R C)^{11}$. Therefore, blockade of ROR $\gamma t$ activity may constitute an alternative to antibody-mediated cytokine and/ or receptor neutralization in treating IL-17-related diseases with a potentially broader mode of action ${ }^{12}$. In addition to conventional Th17 cells, other immune cells showing an innate(-like) phenotype, such as innate lymphoid cells (ILC), subsets of $\gamma \delta$-T cells, mucosal associated invariant T (MAIT) cells and invariant natural killer T cells (iNKT) also express ROR $\gamma t$ and IL-23 signature cytokines $^{13}$. Sherlock et al. showed that a specific subset of CD4CD8-unconventional T cells in mice (which are most likely $\gamma \delta$-T cells) was able to drive SpA-like pathology upon induced systemic overexpression of IL-23, independent of Th17 cells ${ }^{14}$. This suggested that innate-like $\mathrm{T}$ cells may influence diseases like $\mathrm{SpA}^{15}$, because they display plasticity, and could therefore be skewed from an immunoprotective role towards a predominant IL-17 producing phenotype due to uncontrolled IL-23 signaling events. iNKT and $\gamma \delta$-T cells are subpopulations of innate-like T cells, which can release a broad spectrum of cytokines, both upon $\mathrm{T}$ cell receptor (TCR) activation and after TCR-independent stimulation, in this way acting as a 'bridge' between innate and adaptive immune responses. iNKT cells are characterized by the expression of a semi-invariant TCR (invariant Va24-Ja18 in humans) associated with a restricted set of $\mathrm{V} \beta$ chains $(\mathrm{V} \beta 11)$ and they respond towards glycolipids, including microbial $\alpha$ glycuronylceramides and self-antigens, presented by the nonpolymorphic MHC class I-like molecule CD1d ${ }^{16}$. iNKT cells can be separated into three distinctive subsets, iNKT1 cells, iNKT2 cells, and iNKT17 cells, analogous to the helper T cell lineage nomenclature ${ }^{17} \cdot \gamma \delta$-T cells show no distinct MHC class restriction and are reactive towards both peptide (e.g., $\mathrm{MHC}$ related $\mathrm{Ag}$ such as MICA) and non-peptide (e.g., isoprenoid metabolites) antigens, by which they act as sensors of cellular stress and infection danger ${ }^{18}$. Moreover, they appear to be major IL-17 producers in the initial phase of many bacterial infections $\mathrm{s}^{19,20}$. How human innate-like T cells such as iNKT and $\gamma \delta$-T cells are involved in inflammatory responses in arthritic disease, particularly SpA, is unclear and whether these cells can be skewed by pharmacological modulation is equally unknown.

Here we identify novel innate-like $\mathrm{T}$ cell subset-ROR $\gamma \mathrm{t}^{+} \mathrm{T}$ bet $^{\text {loPLZF }}{ }^{-}$iNKT and $\gamma \delta$-hi T cells-circulating in healthy peripheral blood and clearly expanded in inflamed joints, posed to rapidly respond to IL-23 by steering Th17-like immune responses. By using FlowSOM ${ }^{21}$, we show a previously unappreciated heterogeneity of human iNKT and $\gamma \delta$ T cells of which specific subsets are abnormally represented in the peripheral blood of SpA patients. RNASeq analyses revealed a markedly distinct gene signature profile consistent with bias towards distinct IL-17 pathway regulation in innate-like $\mathrm{T}$ cells from $\mathrm{SpA}$ vs. RA patients. Finally, pharmacologic modulation using ROR $\gamma t$ inhibition blocked effector function of disease associated subsets while preserving IL-22 subsets, underscoring the potential of ROR $\gamma t$ antagonism to modulate aberrant type 17 responses.

\section{Results and discussion}

Human blood ROR $\gamma \mathrm{t}^{+}$iNKT and $\gamma \delta$ cells lack expression of PLZF. Murine ROR $\gamma \mathrm{t}^{+}$IL-17 producing iNKT (also denominated iNKT17) and $\gamma \delta$-T cells $(\gamma \delta 17)$ have been extensively studied but the prevalence and phenotype of their human counterparts is poorly understood. We could delineate rare subsets of ROR $\gamma \mathrm{t}^{+}{ }^{\text {T-bet }}{ }^{\mathrm{lo}}$ iNKT $(6 \mathrm{~B} 11+)$ and $\gamma \delta$-T cells in the blood of healthy individuals (Fig. 1a and Supplementary Fig. 1A). ROR $\mathrm{t}^{+}{ }^{+} \mathrm{T}_{\text {-bet }}{ }^{\mathrm{lo}}$ cells represented on average $2.1 \%(0.09-6 \%)$ of total iNKT cells, comparable to frequencies of ROR $\gamma \mathrm{t}^{+} \mathrm{T}$-bet ${ }^{\mathrm{lo}}$ cells $(1.9 \pm 1.4 \%$; mean \pm SEM \%) detected in CD161 + Tconv cells, a population enriched in Th17 cells ${ }^{22}$. The prevalence of ROR $\gamma \mathrm{t}^{+} \mathrm{T}$-bet $\mathrm{t}^{\mathrm{lo}}$ in the $\gamma \delta$-T population was less frequent $(0.5 \pm$ $0.4 \%$ ), but still relatively increased compared to kindred cells in the CD161- Tconv population (Fig. 1b). Interestingly, based on TCR/CD3 expression levels, we could distinguish two populations of $\gamma \delta$-T cells, a large fraction of TCR $\gamma \delta$-int cells and a minor subset of TCR $\gamma \delta$-hi cells, with the latter showing a significant enrichment of ROR $\gamma \mathrm{t}^{+}$T-bet ${ }^{\mathrm{lo}}$ cells (Fig. 1a, b). Phenotypical different subsets of murine $\gamma \delta$-T cells have also been discriminated based on TCR expression levels and/or $\gamma \delta$ gene usage ${ }^{23-25}$. Moreover, $\mathrm{CD} 3^{\text {bright }} \gamma \delta$-T cells showing the canonical $\mathrm{V} \gamma 6 / \mathrm{V} \delta 1+\mathrm{TCR}$ have been correlated with the $\gamma \delta 17$ phenotype and these cells are preferentially found in lung and skin tissues of mice ${ }^{25}$. With regard to TCR gene usage/TCR- $\delta$ repertoire of human $\gamma \delta$ subsets shown here, it was clear that the majority of TCR $\gamma \delta$-int cells belonged to the $\delta 2$ TCR subfamily (being $\mathrm{V} \gamma 9 \mathrm{~V} \delta 2$ ), whereas $\mathrm{TCR} \gamma \delta$-hi cells are enriched in $\delta 1$ cells although they also included a minor subset of $\delta 3$ cells (Fig. 1c and Supplementary Fig. 1B). The capacity of iNKT and these $\gamma \delta$-T cells to secrete IL-17 and to a lesser extent IL-22 cytokines (directly ex-vivo after short term PMA/CaI stimulation), was consistent to their ROR $\gamma t$ expression profile (Fig. 1d and Supplementary Fig. 1C).

In contrast to total iNKT and TCR $\gamma \delta$-int T cells, ROR $\gamma \mathrm{t}^{+} \mathrm{T}$ bet ${ }^{\text {lo }}$ iNKT and TCR $\gamma \delta$-hi T cells showed very low levels of promyelocytic leukemia zinc finger (PLZF) (Fig. 1e, f and Supplementary Fig. 1D). These findings could also be confirmed when using a GalCer-hCD1d tetramers for detection of iNKT cells (Supplementary Fig. 1e, f). In mouse models it has been shown that PLZF plays an important role in the early development of iNKT cells ${ }^{26,27}$ and PLZF expression has also been identified in MAIT cells ${ }^{28}$, innate subsets of $\gamma \delta \mathrm{T}^{\mathrm{T}}$ cells $^{29}$ and fetal $\mathrm{ILC}^{30}$, suggesting a determinant role for PLZF in the common innate phenotype of these cells. In humans, also NK cells and small 


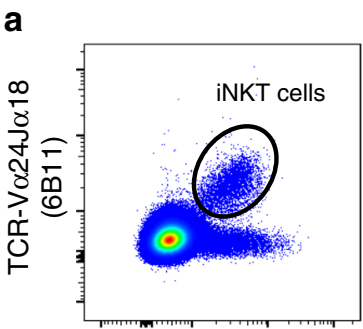

TCR-V $\beta 11$

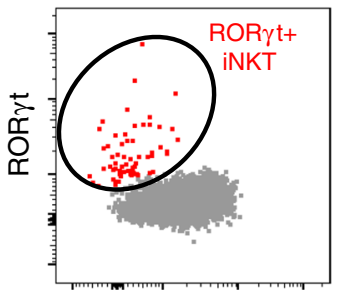

T-bet

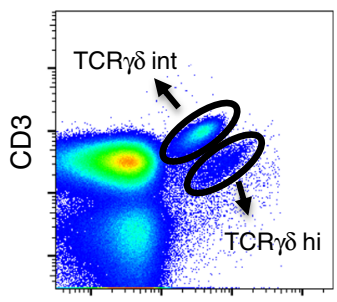

TCR- $\gamma \delta$

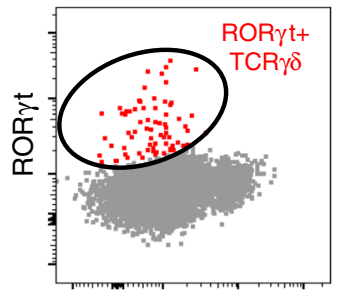

T-bet d
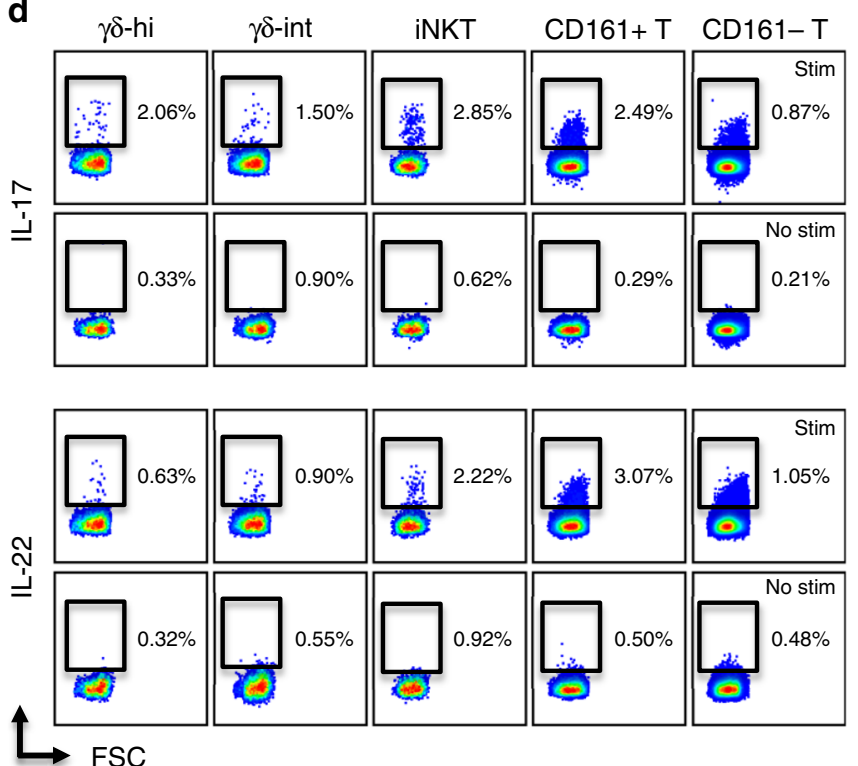

b
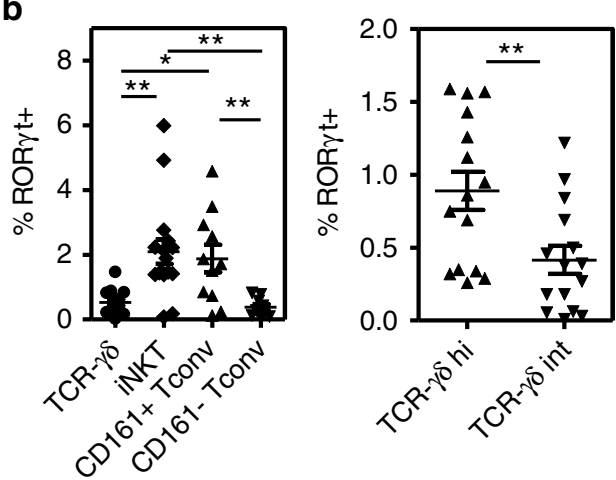

e

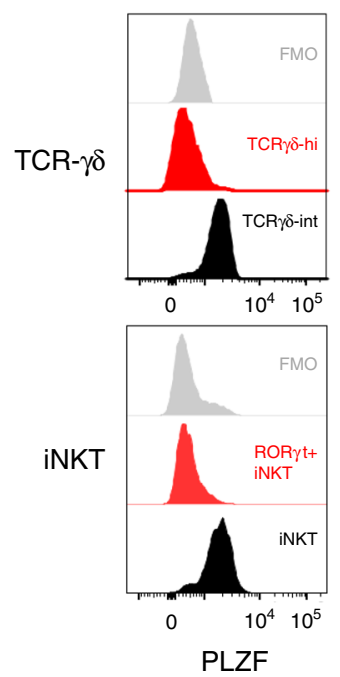

C

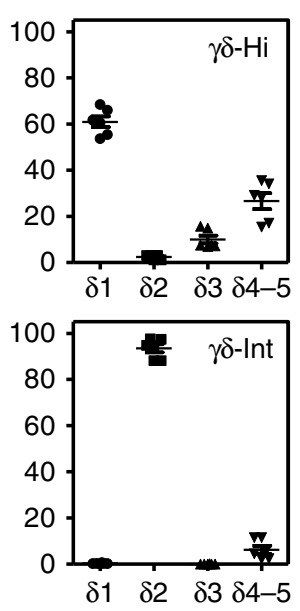

$\mathbf{f}$
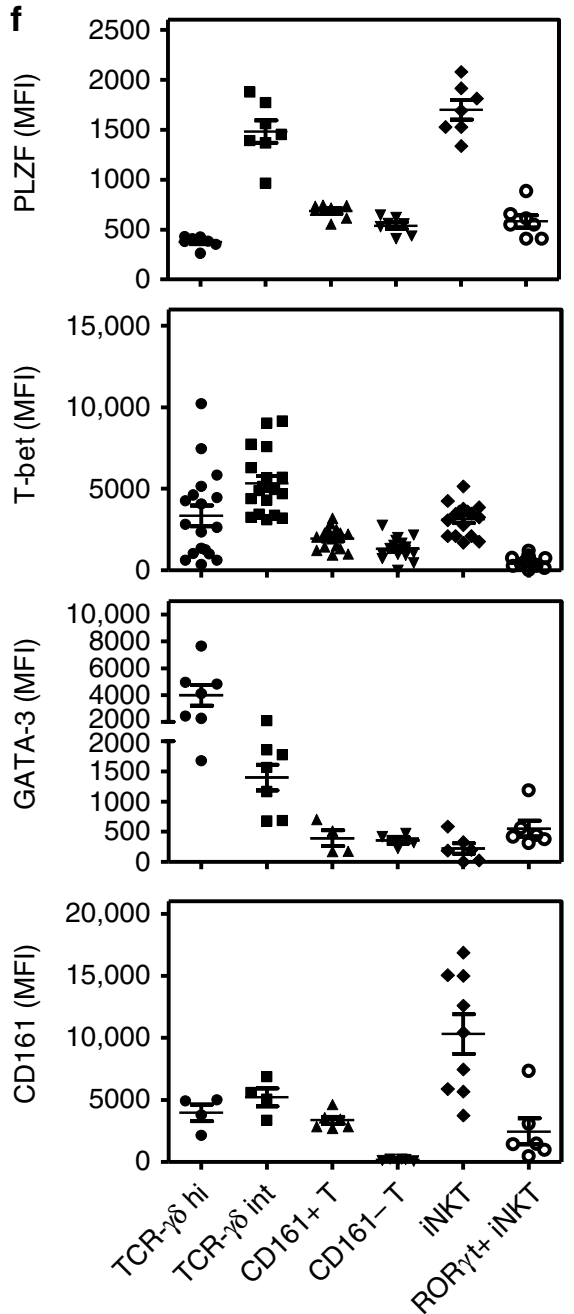

subsets of CD4 and CD8 T cells seem to express PLZF ${ }^{31}$, highlighting substantial differences in the importance of PLZF gene expression between mice and humans. The low PLZF expression in human blood iNKT17 cells seems to contrast observations that PLZF regulates the acquisition and maintenance of a human Th17 phenotype ${ }^{32}$. However, recent studies showed
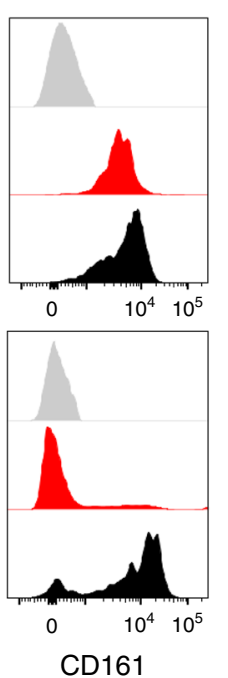

that iNKT cells have a unique transcriptional profile distinct from that of NK cells and MHC-restricted T cells ${ }^{33}$. Moreover, the transcriptional nature of iNKT17 cells was similar to analogous subsets of $\gamma \delta$-T cells and ILC cells, while not to Th17 cells ${ }^{34}$. A possible explanation could be that human ROR $\gamma \mathrm{t}^{+}$iNKT cells develop as a distinct subset of PLZFneg cells in the thymus. At 
Fig. 1 Human blood circulating RORyt+ iNKT and $\gamma \delta-T$ cells lack expression of PLZF. a Flowcytometric analyses showing distinct subsets of blood circulating ROR $\gamma \mathrm{t}^{+} \mathrm{T}$-bet ${ }^{\mathrm{lo}} \mathrm{iNKT}$ and total $\gamma \delta-\mathrm{T}$ cells (flow plots show representative data from a healthy individual). Gating strategy is shown in Supplementary Fig. 1A. b Percentages of ROR $\gamma \mathbf{t}^{+}$T-betlo cells in iNKT $(n=16)$ and $\gamma \delta$-T cells $(n=15)$ and CD161+ and CD161- Tconv ( $\left.n=11\right)$ (left panel; ${ }^{\star} p<0.05,{ }^{\star \star} p<0.01$ as determined by ANOVA) and in $\gamma \delta$-T cell subsets (right panel; paired $t$-test). c TCRV $\delta$ profiling of indicated $\gamma \delta$-T cells ( $n=6$ ). Representative flow plots are shown in Supplementary Fig. 1B (d) IL-17 and IL-22 production by indicated subsets as measured by intracellular cytokine staining after $4 \mathrm{~h}$ incubation of PBMC with PMA/Cal/BFA (stim) or BFA alone (no stim). Results from one experiment are shown (representative for $n=6$ ). Summary data are presented in Supplementary Fig. 1C. e Stacked histograms showing expression levels of PLZF, T-bet, GATA-3, and CD161 measured in iNKT and $\gamma \delta$-T cells (one representative example). $\mathbf{f ~ M F I ~ v a l u e s ~ f o r ~ i n d i c a t e d ~ m a r k e r s ~ ( a s ~ i n d i c a t e d ~ i n ~} \mathbf{d}$ ). Each dot represents data from one healthy individual (PLZF and GATA-3 $n=7 ;$ CD161 $n=9$, T-bet $n=16$ ). Overview of statistics (ANOVA) is provided in the Supplementary Table 3. Data throughout this figure are presented as mean \pm SEM

least in mice this scenario seems unlikely, as thymic iNKT17 cells express intermediate levels of PLZF, in between those measured in iNKT1 $\left(\mathrm{PLZF}^{\mathrm{lo}}\right)$ and iNKT2 (PLZFhi) cells ${ }^{17}$. Alternatively, iNKT17 cells might lose PLZF expression upon peripheral migration and/or activation and differentiation. In this regard, PLZFlo iNKT cells have been identified in murine adipose tissue, not linked to ROR $\gamma \mathrm{t}$ expression or IL-17 production but to an anti-inflammatory IL-10 driven phenotype, indicating a specific local role for these PLZFlo iNKT cells in adipose tissue immunoregulation $^{35}$. In mice, iNKT17 cells are very scarce in peripheral circulation and the majority of iNKT17 cells seem to be tissue resident cells that can be found in lung tissue and lymph nodes $^{36}$. iNKT subsets are uniquely distributed in peripheral lymphoid and non-lymphoid organs with some inter-strain variation $^{36}$. They do not (or only modestly) recirculate, but instead show very long-term residence at these locations ${ }^{35,37}$. Our data suggest that human peripheral blood ROR $\gamma \mathrm{t}^{+} \mathrm{T}$-bet ${ }^{\mathrm{lo}}$ iNKT cells might show an intermediate/transitional phenotype of iNKT17 cells, prior to migration of these cells into various tissues, where they will further differentiate under influence of the local microenvironment and signaling cues.

Most work on human $\gamma \delta$-T cells has focused on $\mathrm{V} \gamma 9 \mathrm{~V} \delta 2$ $\mathrm{T}$ cells, which form the major population of blood $\gamma \delta$-T cells. Of note, this subset is not found in mice due to the absence of orthologous TCR gene segments ${ }^{18}$. We found that $\gamma \delta$-T cells contain two phenotypically distinct subsets based on TCR expression levels, with TCR $\gamma \delta$-hi cells being predominantly $\mathrm{PLZF}^{+}, \mathrm{GATA}^{-}{ }^{+}$, and $\mathrm{Tbet}^{\mathrm{lo}}$. Although the data on the role of PLZF in $\gamma \delta$-T cells are more limited, they also point towards an innate differentiation program in these cells, as noticed for selective subsets of murine $\left(\mathrm{V} \gamma 1^{+} \mathrm{V} \delta 6.3 / \mathrm{V} \delta 6.4^{+}\right) \quad \gamma \delta$-T cells ${ }^{29,31,38}$. In contrast, previous work indicated that PLZF is present in all human $\gamma \delta$-T cells ${ }^{31}$, but our data highlight this is especially true for the TCR $\gamma \delta$-int cell population, while TCR $\gamma \delta$-hi cells, containing augmented numbers of ROR $\gamma \mathrm{t}^{+}$cells (Fig. 1b), are clearly PLZFlo. This seems in concordance with Kreslavsky et al. showing that thymic mouse $\gamma \delta$-T cells capable of producing IL-17 are PLZF negative ${ }^{29}$. However the majority of human PLZFlo $\gamma \delta$-T cells did not express ROR $\gamma \mathrm{t}$, so the precise relation between expression of these transcription factors in $\gamma \delta$-T cells needs further investigation.

Altogether, these data identify ROR $\gamma \mathrm{t}^{+}$iNKT and $\gamma \delta$-hi T cells as a minor fraction of PLZFneg innate-like $\mathrm{T}$ cells in the peripheral blood of healthy individuals.

iNKT and $\gamma \delta$-T cells are armed for a competent IL-23 response. As IL-23 and IL-23 receptor expression is clearly associated with Th17 pathogenicity ${ }^{13}$, we examined IL-23 responsiveness of human iNKT and TCR $\gamma \delta \mathrm{T}$ cell subsets. Consistent with data obtained from IL-23R reporter mice ${ }^{39}$, we noticed that surface IL23R expression was almost completely absent on human blood derived iNKT and TCR $\gamma \delta \mathrm{T}$ cells (Supplementary Fig. 2A). In naïve mice, IL-23R is prominently found on lamina propria lymphocytes (LPL) $\gamma \delta$-T cells ${ }^{39}$, underscoring the importance for IL-23 at barrier sites. Using PrimeFlow technology, we measured low but significant amounts of RORC mRNA transcripts in iNKT, TCR $\gamma \delta$-int, and CD161+ conventional T cells, in contrast to high levels in TCR $\gamma \delta$-hi T cells (Fig. 2a and Supplementary Fig. 2B). Interestingly, significant IL-23 receptor (IL-23R) mRNA transcripts could be found in iNKT cells, a subset of CD161+ T cells and the majority of TCR $\gamma \delta \mathrm{T}$ cells. In addition to differences in $R O R C$ mRNA, Il-23R mRNA was also expressed at higher levels in TCR $\gamma \delta$-hi as compared to TCR $\gamma \delta$-int cells further underscoring the phenotypical variance between these $\gamma \delta$-T cell subsets.

Next, we tested blood TCR $\gamma \delta$ and iNKT cell function in response to IL-23 stimulation in vitro. As expected from the absence of significant surface IL-23R expression, short term stimulation of sorted iNKT or TCR $\gamma \delta$ T cells with IL-23 alone did not lead to IL-17 release by any cell type (data not shown). However, upon culturing iNKT or TCR $\gamma \delta$ T cells with a TCR stimulus (aGalCer or aCD3/CD28Ab, respectively), we found a strong enrichment of IL-17 producing cells in those cultures where we added IL-23 as compared to IL-2 alone (Fig. 2b), in agreement with published data ${ }^{20,40}$. TCR $\gamma \delta$-hi cells showed an augmented capacity to produce IL-17 and also IL-22 as compared to TCR $\gamma \delta$-int cells upon IL-23 priming (Fig. 2b, c). TCR $\gamma \delta$-hi cells producing IFN $\gamma$ and TNFa were also induced by IL-23, matching the frequencies found in TCR $\gamma \delta$-int cells. Parallel to induction of IL-17, IL-23 skewed iNKT cells contained significantly increased numbers of $\mathrm{IL}-21^{+}$cells, whereas the frequency of IL-22, TNFa and IFN $\gamma$ producing cells was comparable between the IL-2 and IL-23 conditions (Fig. 2d). The phenotypical profile of IL-23 induced IL-17+ iNKT cells was further evaluated by means of flow cytometry and qPCR analyses on sorted IL-17 ${ }^{+}$cells as obtained by a cytokine capture assay (Fig. 2e, f). These data confirmed that IL-23 induced iNKT17 cells showed higher levels of RORC and lower levels of T-bet expression and CD161 as compared to IL-17 ${ }^{\text {neg }}$ counterparts (Fig. 2e and Supplementary Fig. 2C), mimicking the ex-vivo phenotype (Fig. 1d). Moreover, iNKT17 cells also expressed higher levels of CCR6 a chemokine receptor associated with Th17 cells, and contained high levels of IL17A, IL17F, and also IL22 mRNA (Fig. 2f).

Overall, these data indicate that human circulating iNKT cells and especially $\gamma \delta$-hi T cells show significant levels of IL $23 R$ mRNA transcripts which enables them to rapidly induce Th17-like immune responses.

iNKT and $\gamma \delta$-T cells in SpA joints show a IL-17 signature. Because recent data point to the importance of IL-23/IL-17 driven inflammation in mediating disease features in SpA, we examined ROR $\gamma \mathrm{t}^{+}$innate-like T cells in blood and synovial fluid (SF) from new onset SpA patients. We first analyzed overall frequencies of iNKT and TCR $\gamma \delta$ T cell subsets in paired peripheral blood and SF samples from patients with active joint swelling. The frequency of blood circulating iNKT cells in SpA was significantly reduced vs. 
a

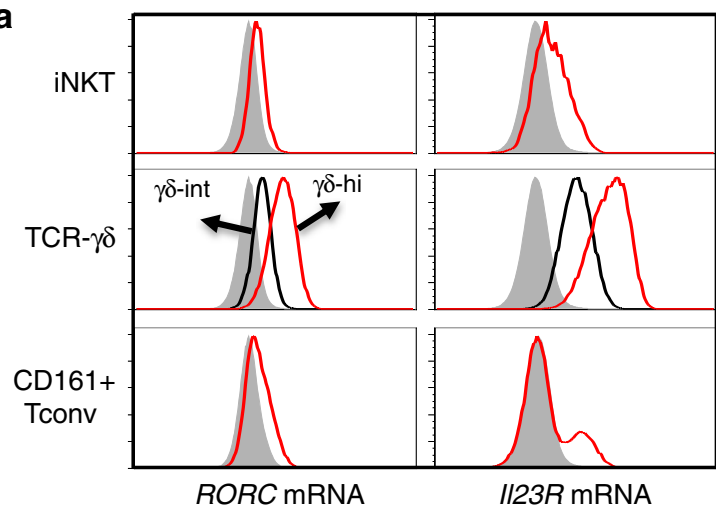

b e

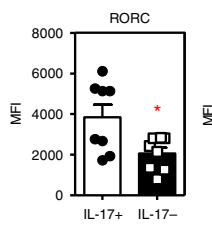

f

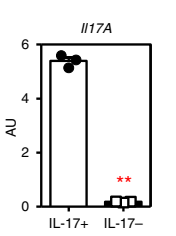

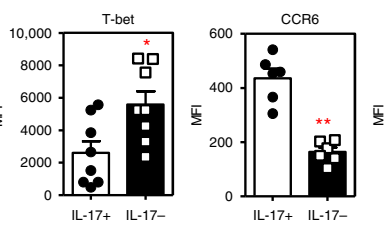
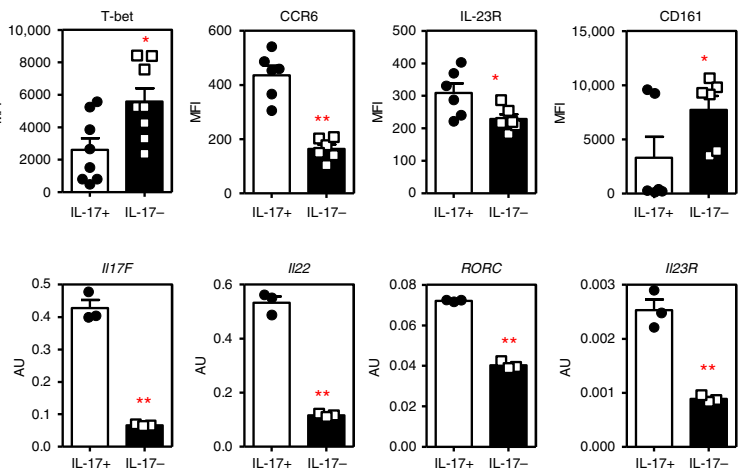

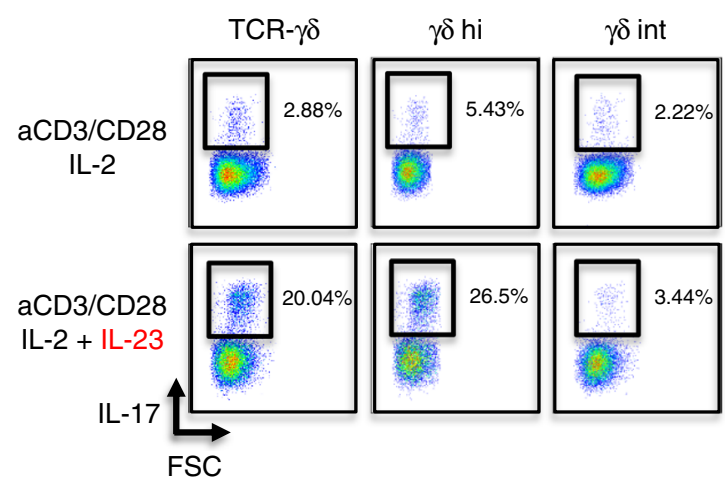

C
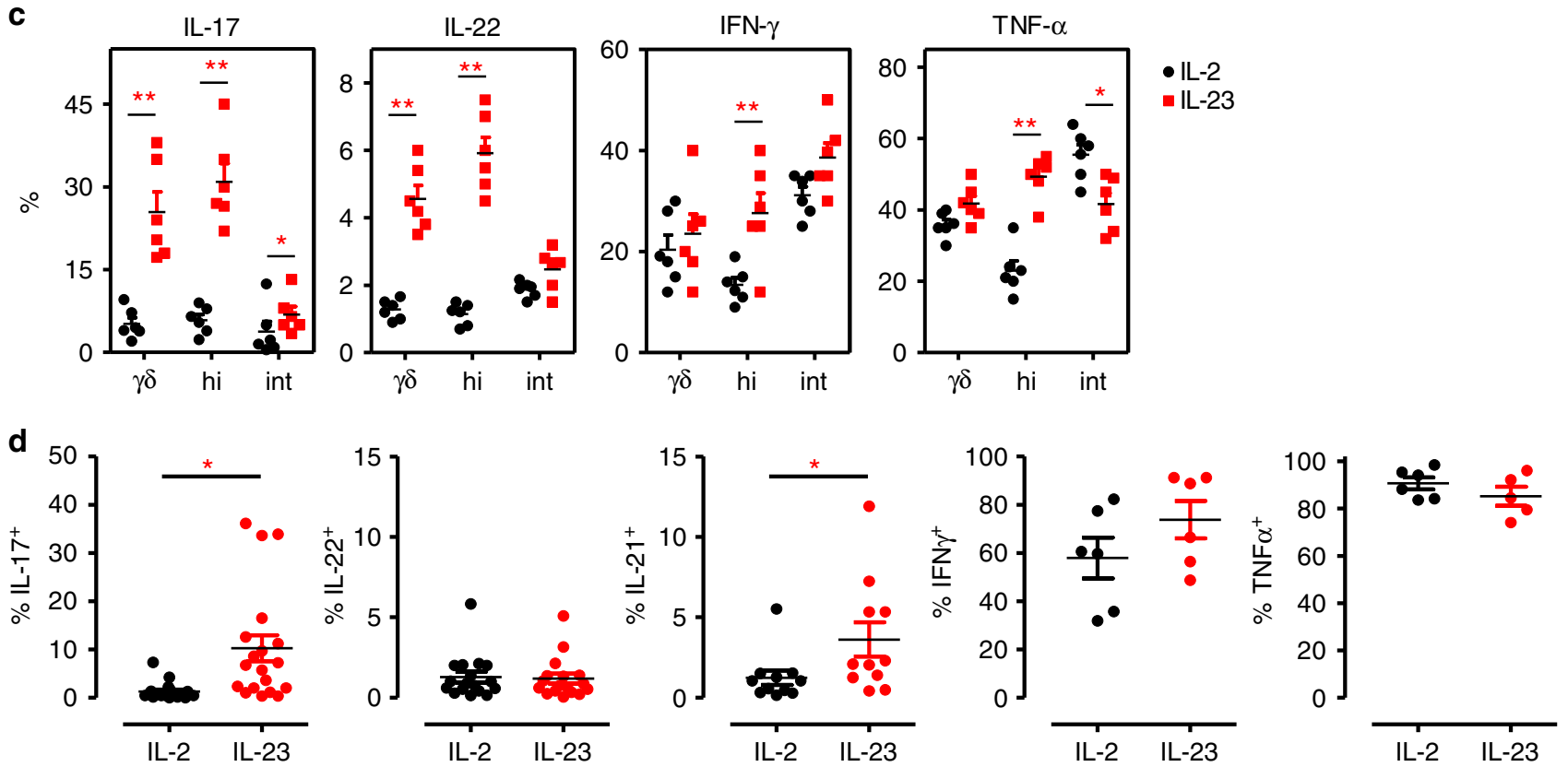

Fig. 2 iNKT and $\gamma \delta$-T cells are armed for a competent IL-23 response. a RORC and IL23R mRNA transcripts expressed in iNKT, TCR $\gamma \delta$-hi/int, and CD161+ conventional T cells as measured by PrimeFlow technology $(n=3)$. Gray histograms represent FMO. Quantitative data are presented in Supplementary Fig. 2B. b PBMC (left) or sorted $\gamma \delta$-T cells were cultured with $\alpha$ GalCer (PBMC, $n=18$ ) or aCD3/aCD28 Abs $(\gamma \delta$-T cells, $n=6)$, in the presence of IL-2 or IL-2 supplemented with IL-23, IL1 $\beta$, TGF $\beta 1$ to induce IL-17 cytokine response as measured from viable iNKT ( $6 \mathrm{~B} 11+\mathrm{TCRv} \beta 11+\mathrm{CD} 3+$ cells) or $\gamma \delta$-T cells $(\mathrm{TCR} \gamma \delta+\mathrm{CD} 3+)$. Flow data from one representative experiment is shown. $\mathbf{c}$, $\mathbf{d}$ Quantitative overview of cytokine production measured in $\gamma \delta-\mathrm{T}$ cell (C; IL-2: black symbols; IL-23: red symbols) and iNKT (D, IL-2 condition: black symbols; IL-23: red symbols) cultures as described in b (IL-23 vs. IL-2 condition, ${ }^{\star} p<$ $0.05,{ }^{\star \star} p<0.01$ determined by paired $t$-tests). e FACS analyses were performed on gated IL17+ and IL17- iNKT cells depicted in experiments described in b $(n=6-8) .{ }^{\star} p<0.05,{ }^{\star \star} p<0.01$ determined by paired t-tests (IL-17- cells: black bars; IL-17+: white bars). f qPCR assays on selected target genes, were performed on IL17+ and IL-17- iNKT cells isolated by means of a IL-17 Capture Assay ( (PPCR, $n=3$, Mann-Whitney test). (IL-17- cells: black bars; IL-17+: white bars). Data throughout this figure are presented as mean \pm SEM 
a

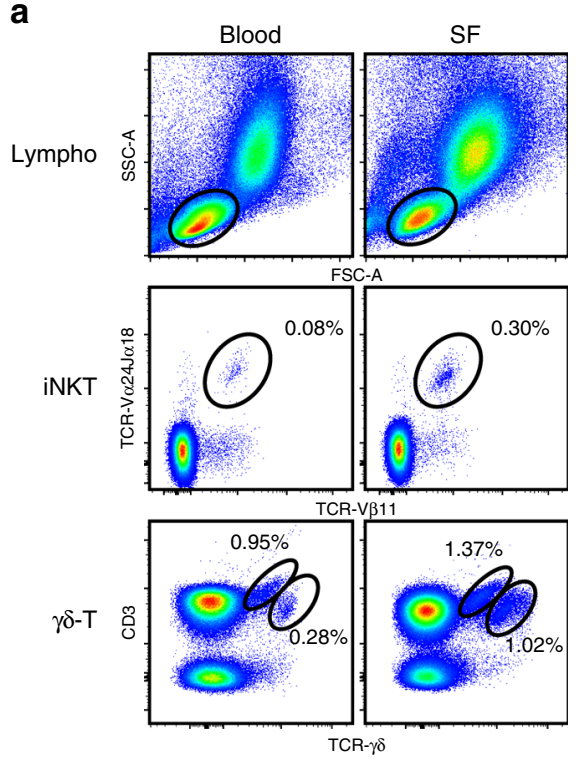

e
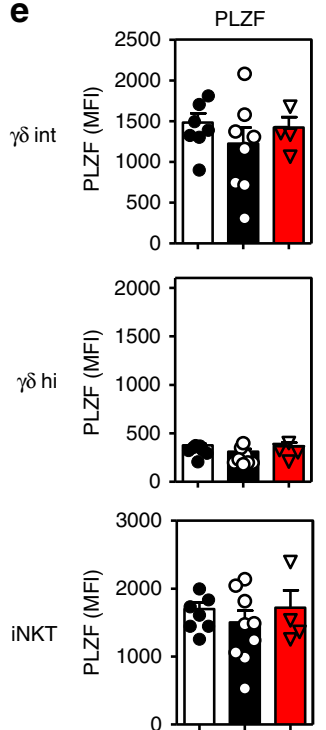
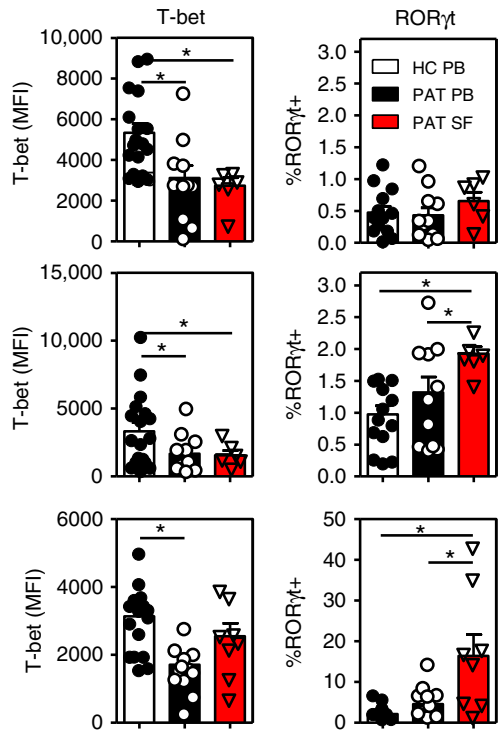

b

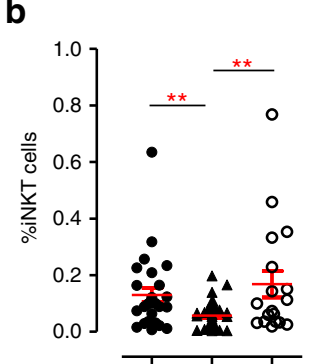

d

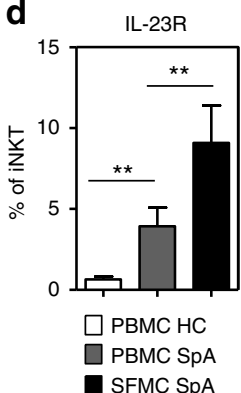

C
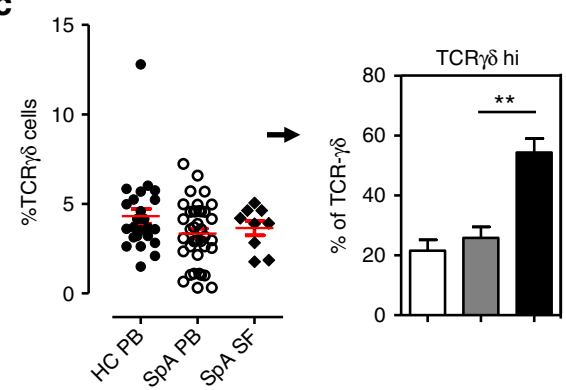

f iNKT cells
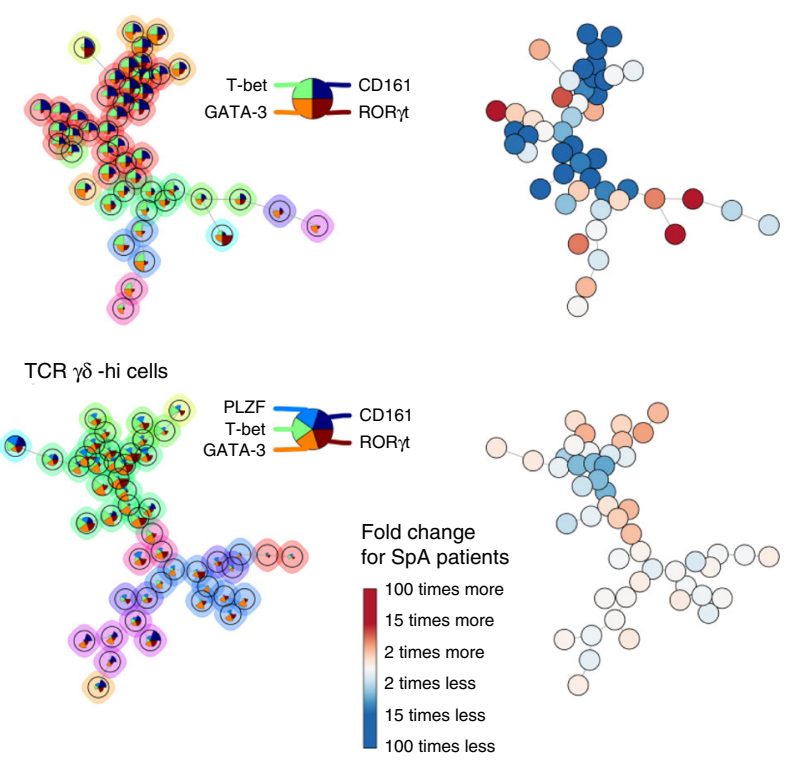

g
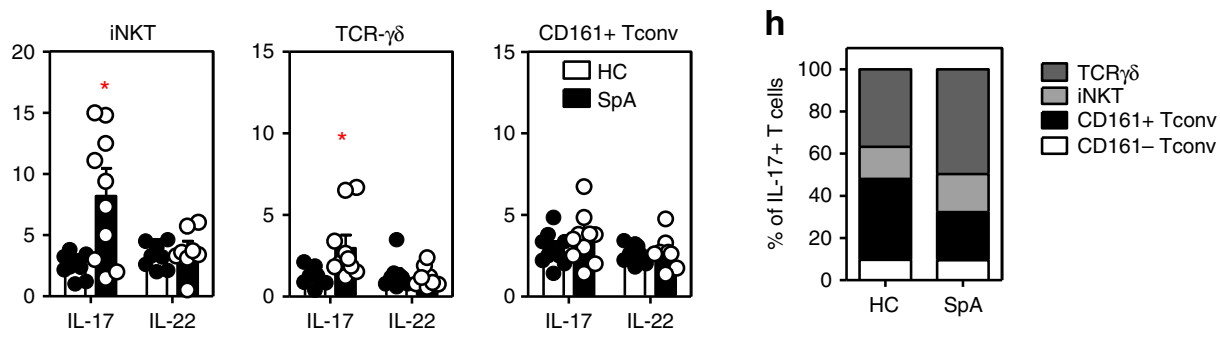

age matched healthy controls (Fig. 3a, b; HC $0.130 \pm 0.025 \%$ vs. SpA $0.051 \pm 0.008 \%$; $p<0.01$, ANOVA). By contrast, iNKT cell numbers were increased in the SF samples of these patients $(0.168 \pm 0.047 \% ; p<0.01$ as compared to SpA blood). Frequencies of $\gamma \delta$-T cells were not significantly reduced in circulation of SpA patients compared to healthy controls (Fig. 3c; HC $4.31 \pm 0.41 \%$ vs. SpA $3.34 \pm 0.27 \%$; $p>0.05$, ANOVA). But curiously, in SF of SpA patients the prevalence of especially the TCR $\gamma \delta$-hi subset was remarkable augmented compared to peripheral blood (Fig. 3c; SpA blood $25.8 \pm 3.6 \%$ vs. SF $54.3 \pm 4.7 \%$ of $\gamma \delta$ cells; $p<0.01$,
ANOVA). These alterations in iNKT and $\gamma \delta$-T cells were also reflected in patient derived synovial tissue samples (inflamed knee) (Supplementary Fig. 3). Moreover, there was also a significant increase in the number of IL-23R ${ }^{+}$iNKT and $\gamma \delta$-T cells in the joint compartment of SpA patients (Fig. 3d; iNKT: blood $4.3 \pm 1.2 \%$ vs. SF $9.0 \pm 2.2 \%$; Fig. $5 \mathrm{c} \gamma \delta$-T: blood $4.6 \pm 1.7 \%$ vs. SF $17.9 \pm 13.9 \%$ of $\gamma \delta$-T cells; both $p<0.01$, ANOVA).

A hallmark of innate-like T cells such as iNKT and $\gamma \delta$-T cells is their functional plasticity which is reminiscent, and may even exceed, that of conventional CD4 T cells ${ }^{3}$. We therefore analyzed 
Fig. 3 iNKT and $\gamma \delta$-T cells in SpA joints show a profound IL-17 signature. a Flowcytometric analyses of iNKT and $\gamma \delta-T$ cells in paired PBMC and SFMC derived from a representative SpA patient. b Mean percentage ( \pm SEM) of iNKT cells (relative to total CD3+ T cells) measured in PBMC (SpA, $n=33$; HC, $n=27)$ and SFMC $(S p A, n=18)$ samples $\left({ }^{\star \star} p<0.01\right.$ as determined by ANOVA). c Frequency of $\gamma \delta$-T cells and TCR $\gamma \delta$-hi cells (relative to total CD3+ T cells and $\gamma \delta$-T cells, respectively) in PBMC and SFMC samples (ANOVA). $\mathbf{d}$ Mean percentage IL-23R+ cells (relative to iNKT cells) measured in PBMC and SFMC samples from B (ANOVA). e Transcriptional factor profile of iNKT cells and $\gamma \delta-T$ cells measured in PBMC and SFMC from SpA patients (PB $n=$ 8-12, SF $n=4-8)$ and HC ( $n=8-16)$. ( ${ }^{*} p<0.05$ as determined by ANOVA) (HC-PB: white bars, SpA-PB: black bars, SpA-SF: Red bars). $\mathbf{f}$ FlowSOM visualization of indicated immune cell population present in the peripheral blood of $\mathrm{HC}(n=8)$ and SpA patients $(n=8)$, stained for markers indicated in the pie chart. Each circle represents a specific marker combination, corresponding to a specific cell type. The mean marker values are visualized for each node, using star charts. The height of each part indicates the expression intensity: if the part reaches the border of the circle, the cells have a high expression for that marker. The nodes are connected to the ones they are the most similar to. An automatic meta-clustering of the FlowSOM nodes is visualized by the background color of groups of nodes. A parallel tree gives an overview of significantly over-(red) or under-(blue) represented cell types in SpA patients vs. controls. $\mathbf{g}$ IL-17 and IL-22 production by peripheral blood iNKT and $\gamma \delta$-T cells from SpA patients $(n=10 ;$ black bars) and controls $(n=10$, white bars) as measured by intracellular cytokine staining after $4 \mathrm{~h}$ incubation of cells with PMA/Cal in the presence of BFA ( ${ }^{*} p<0.05, t$-tests $\mathrm{HC}$ vs. $\mathrm{SpA}$ ). $\mathbf{h}$ Relative representation of indicated peripheral blood T cell subsets among IL-17 producing T cells as measured in PMA/Cal stimulated PBMC from $\mathrm{HC}$ and SpA patients (see Supplementary Fig. 6A for individual data points and statistics). Data throughout this figure are presented as mean \pm SEM unless stated otherwise

the transcription factors controlling effector functions in these subsets in the blood and joints of SpA patients. ROR $\gamma \mathrm{t}^{+}$cells in iNKT and to lesser extent TCR $\gamma \delta$-hi, were significantly enriched in SF vs. blood and accompanied with a decrease in T-bet levels. T-bet levels were also significantly reduced in circulating iNKT cells and $\gamma \delta$-T cells of SpA patients compared to controls (Fig. 3e). In contrast, no significant differences were found in PLZF expression (Fig. 3e). The flow cytometry dataset generated from the patient and control samples was further evaluated by FlowSOM, a novel and highly efficient tool for unsupervised clustering of cell types ${ }^{21}$. Traditional, linear analyses of multiparameter flow cytometry data can suffer from limitations and the recent progress in computational methods has led to novel insights into immune cell diversity ${ }^{41}$. Human peripheral blood iNKT and $\gamma \delta$-T cell subsets were visually represented in a minimal spanning tree, where each node (circle) corresponds to a group of phenotypically related cells, and neighboring circles represent more similar cell types (Fig. 3f). FlowSOM metacluster analyses showed a marked heterogeneity of blood circulating iNKT and $\gamma \delta$-T cell subsets (based on their transcriptional factor profile). Moreover, we observed a skewing of particular innatelike $\mathrm{T}$ cell subsets in the blood of SpA patients, which was most pronounced for iNKT cells (Fig. 3f and Supplementary Fig. 4). SpA patient blood circulating conventional $\mathrm{T}$ cells showed a comparable FlowSOM profile with corresponding cells from control individuals, although some minor significant differences were observed for the CD161+ population (Supplementary Fig. 4 and 5). When we evaluated their cytokine production (directly ex-vivo after short term PMA/CaI stimulation), we observed increased numbers of $\mathrm{IL}-17^{+}$cells in blood derived iNKT and $\mathrm{TCR} \gamma \delta$ but not Tconv cells from SpA patient vs. healthy controls (Fig. 3g; iNKT $2.81 \pm 0.64 \%$ vs. $8.20 \pm 2.27 \% \mathrm{IL}^{-17^{+}} ; \gamma \delta: 1.16 \pm$ $0.21 \%$ vs. $2.97 \pm 0.80 \% \mathrm{IL}^{-}-17^{+}$for $\mathrm{HC}$ vs. SpA, respectively; both $p<0.05$, ANOVA). Moreover, innate-like T cells represent about half of all IL-17 producing blood circulating $\mathrm{T}$ cells, and this fraction is even higher in SpA (Fig. 3h and Supplementary Fig. 6A).

iNKT and $\gamma \delta$-T cells were found to be even further skewed towards ROR $\gamma \mathrm{t}$ and IL-17 expressing subsets in SF samples from SpA patients (Fig. 4a, b) as determined by FLOWSOM (Fig. 4a) and intracellular cytokine stainings (Fig. 4b). Moreover, elevated ROR $\gamma$ t expression levels were also measured in synovial tissue residing iNKT and $\gamma \delta$-T cells (Supplementary Fig. 3). To assess the contribution of these innate-like T cells in IL-17 inflammation in the joints, we selectively depleted iNKT and $\gamma \delta$-T cells from SpA SF mononuclear cells (SFMC) and assessed the impact on cytokine production following TCR ligation. For this, we measured IL-17 and IL-22 (next to TNF $\alpha$ and IFN $\gamma$ ) secretion in cultures of SFMC in the presence or absence of iNKT and $\mathrm{TCR} \gamma \delta$ cells (SFMC and $\triangle$ SFMC, respectively). Both SFMC and $\triangle$ SFMC fractions were processed under identical conditions and stimulated with or without IL-23 and in the absence or presence of TCR stimulation (Fig. 4c). These experiments highlighted that the absence of iNKT/TCR $\gamma \delta$ cells led to a marked decrease in IL17, IL-22, and IFN $\gamma$ production upon TCR ligation, a finding also observed under different stimulation conditions (Supplementary Fig. 6B). TNFa levels did not significantly change in $\triangle \mathrm{SFMC}$ vs. SFMC cells (Fig. 4c). Interestingly, IL-23 alone also induced some IL-17 in SFMC but not in $\triangle$ SFMC cells (Fig. 4c) consistent with the observed IL-23R abundance on these cells in the joint compartment. These functional data provide clear evidence that innate-like $\mathrm{T}$ cells such as iNKT and $\gamma \delta$-T cells, although numerically not the most abundant immune cell subset, act as major T cell sources of IL-17, IL-22, and IFN $\gamma$ in SpA joints.

Taken together, there is a previously unrecognized diversity of human innate-like $\mathrm{T}$ cells of which some ROR $\mathrm{tt}^{+}$subsets show a particular skewing in SpA patient blood and joint fluid. Thus, ROR $\mathrm{t}^{+}$iNKT and $\gamma \delta-\mathrm{T}$ cells accumulate in joints of patients with SpA, and potentially together with other innate-like immune cells such as MAIT ${ }^{42}$ and ILC ${ }^{43,44}$ act as major contributors to IL17 mediated pathology in SpA joints.

iNKT and $\gamma \delta$-T cells show different gene expression profiles in SpA and RA. RA is a chronic autoimmune joint disease which has also been linked to Th17 mediated pathology ${ }^{45}$. Unexpectedly, inhibition of IL-17A with secukinumab, as showed in a phase 3 clinical trial, failed to demonstrate major clinical benefits ${ }^{7}$, which contrast with its broad efficacy in $\mathrm{SpA}^{4}$. We therefore evaluated whether the altered iNKT17 and $\gamma \delta 17 \mathrm{~T}$ cell profile we observed was a specific finding for SpA. We first evaluated iNKT and $\gamma \delta$-T cell numbers in blood and SF samples of treatment naïve RA patients in addition to patients with crystal induced arthritis (CrA, including gout/chondrocalcinosis), prototypic forms of acute -inflammasome/IL-1- driven arthritis ${ }^{46}$ (Fig. 5a). An enrichment of iNKT cells in peripheral joints was also observed in RA patients, but not in CrA patients Fig. 5a). There were no significant differences in total TCR $\gamma \delta+$ cell numbers in blood vs. SF samples from RA nor CrA patient and only SpA patients showed a significant increase in IL-23R $\mathrm{R}^{+} \gamma \delta$-hi cells in the joints (Fig. 5b, c). Notable, there was a subset of IL$23 \mathrm{R}^{+} \gamma \delta$-T cells clearly present in the SF of CrA patients (Fig. $5 \mathrm{c}$ ). This seems unrelated to observations in $\mathrm{SpA}$, as these CrA patient $\gamma \delta$-T cells were not enriched in TCR $\gamma \delta$-hi cells. Whether these 
a

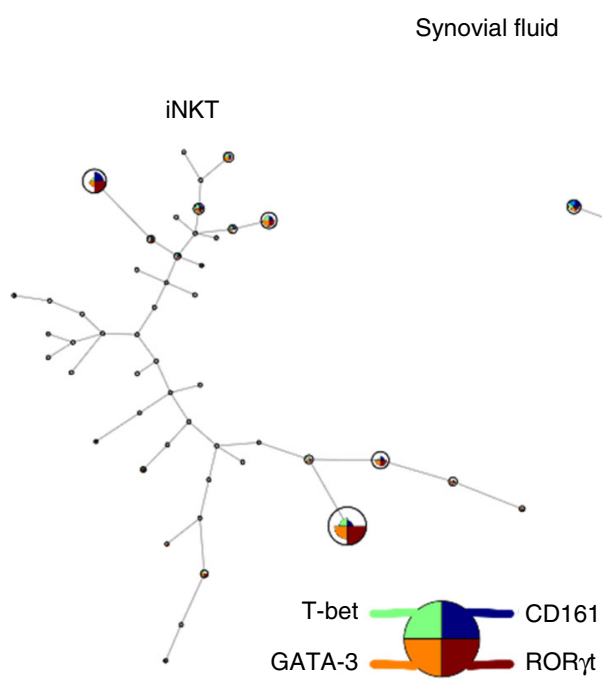

b
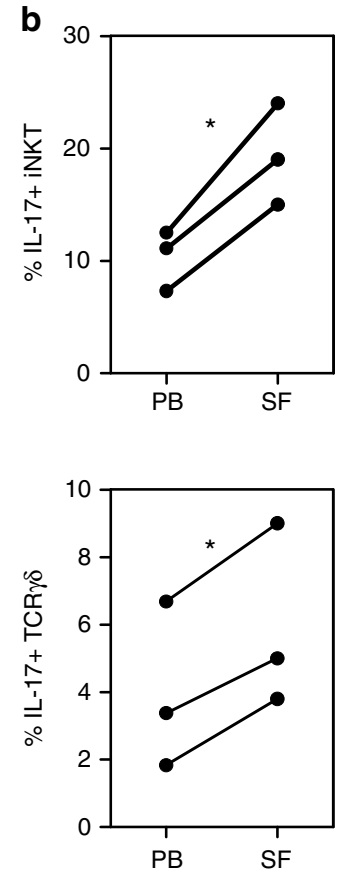

C
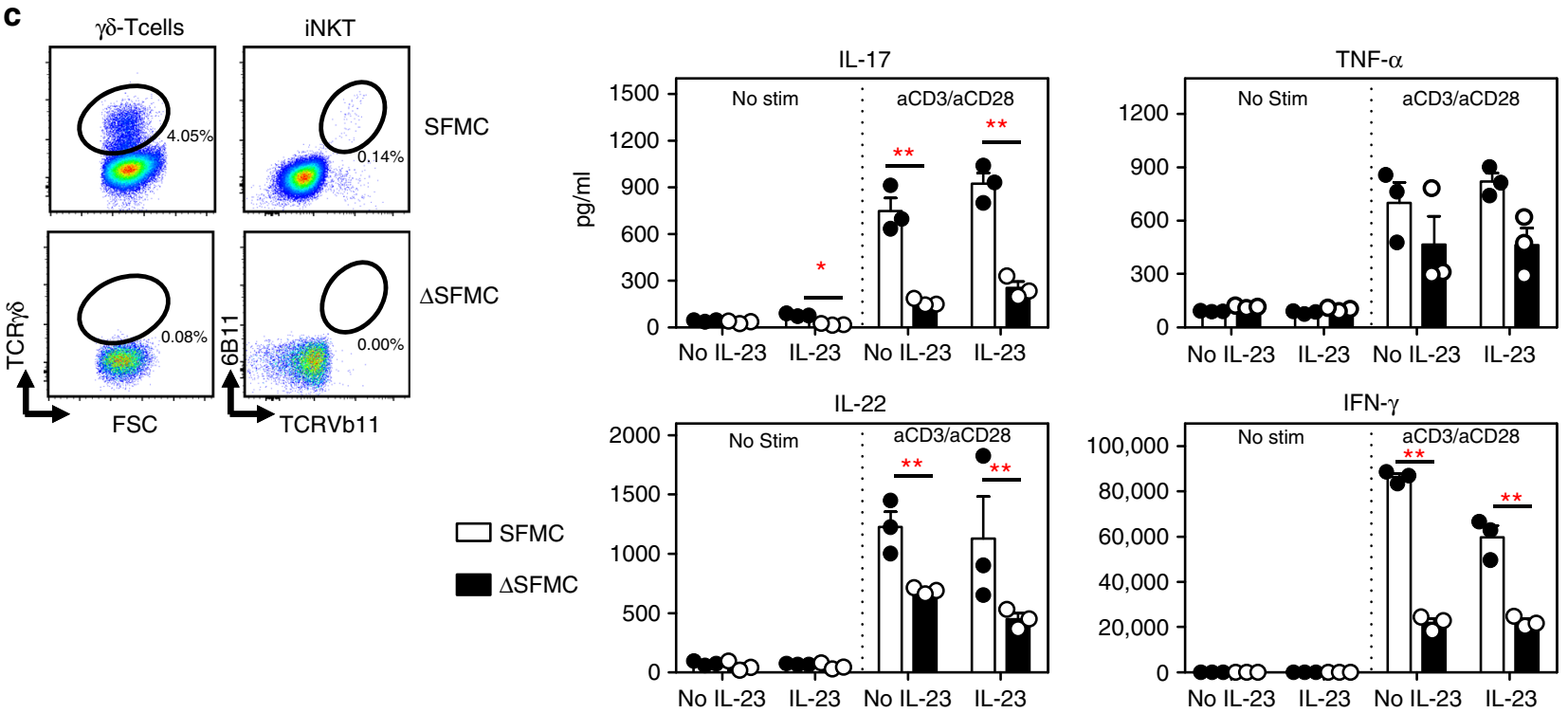

Fig. 4 iNKT and $\gamma \delta$-T cells are a major source of T cell derived IL-17 in SpA joints. a FlowSOM visualization of iNKT and $\gamma \delta$-hi T cells present in synovial fluid samples from three treatment naïve SpA patients, stained for the indicated markers set (as explained in Fig. 3F). Nodes show cell subsets (including ROR $\gamma \mathrm{t}$ + cells) enriched in the synovial fluid (compared to cells from paired blood samples). Other subsets (nodes) are present but at a relatively lower frequencies. b Paired analyses of IL-17 production by SpA SF and blood derived iNKT and $\gamma \delta-T$ cells as determined in Fig. 3G. ( ${ }^{*} p<0.05$, paired $t$-tests). c Synovial fluid derived mononuclear cells (SFMC) and SFMC cells depleted of iNKT and TCR $\gamma \delta$ cells ( $\triangle$ SFMC, illustrated in the plots) were cultured in the presence or absence of IL-23 combined with or without aCD3Ab/aCD28Ab stimulation. Supernatants were collected at $72 \mathrm{~h}$ of culture and cytokines production was measured by ELISA (SFMC vs. $\triangle$ SFMC; ${ }^{\star} p<0.05$, ${ }^{\star \star} p<0.01$ two-way ANOVA). Results from one experiment are shown (Representative for a total of three independent experiments using SF samples from 3 SpA patients). SFMC data (white bars), $\triangle$ SFMC (black bars). Data throughout this figure are presented as mean \pm SEM

cells are potentially driven by uric acid crystal induced IL-1 signaling events needs additional study.

We sorted peripheral blood iNKT and TCR $\gamma \delta$ T cells next to CD161- Tconv cells from $7 \mathrm{SpA}$ and 5 RA patients prior to gene expression profile analyses. Principal component analysis (PCA) of normalized transcriptomics data showed separate clustering of iNKT cells of RA patients and SpA patients and to a lesser extent also for $\gamma \delta$-T cells and Tconv cells indicating clear differences in gene expression profiles between SpA and RA patients (Fig. 5d).
Next, differentially expressed genes in SpA vs. RA derived cells were evaluated and volcano plots were generated showing genes (red dots) at least 2 fold upregulated in either SpA or RA (adjusted $p<0.05$ ) (Fig. 5e). Pairwise comparison of iNKT, $\gamma \delta$-T cells and Tconv of SpA and RA patients indicated 1594, 168, and 1206 , respectively, differentially expressed genes. When comparing cells from SpA and RA patients, we detected no significant differences in expression of the Th cell signature transcription factors, RORC (Th17), TXB21 (Th1) or GATA3 (Th2), for any of 
a
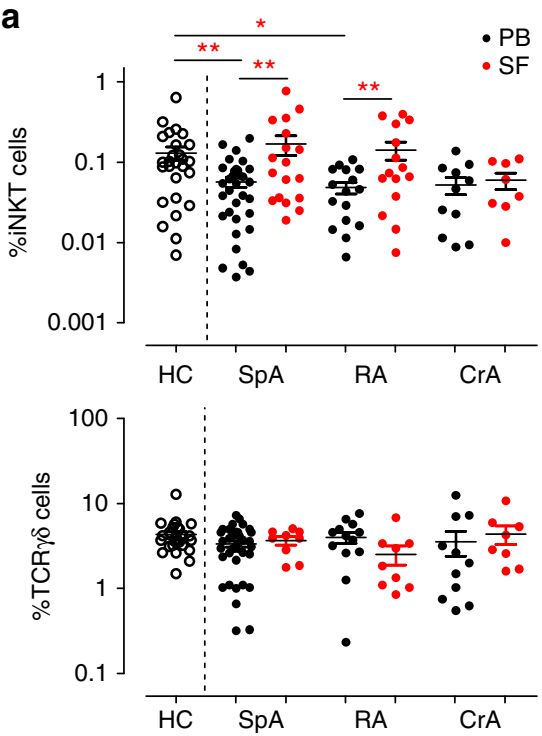

d

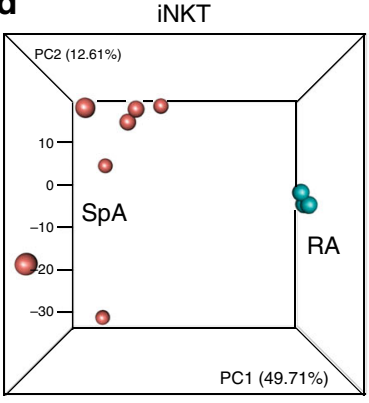

$\gamma \delta \mathrm{T}$
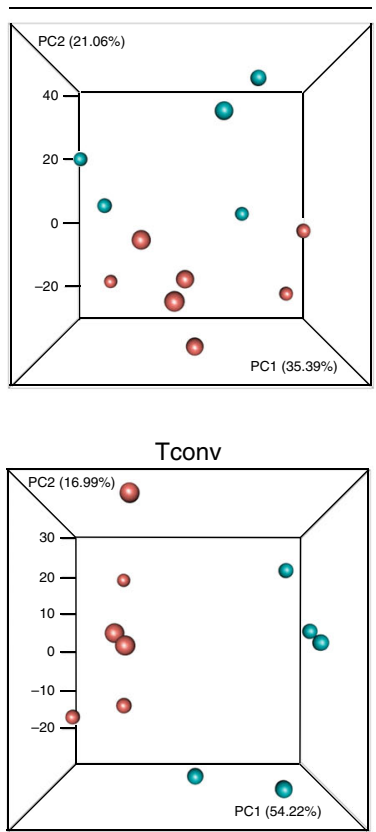

b

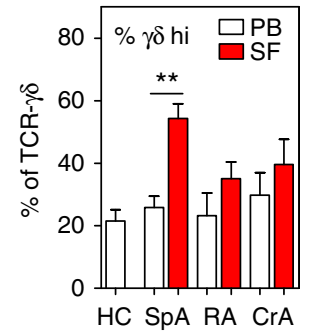

C

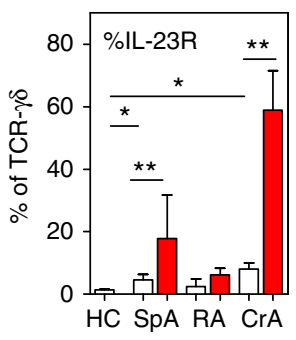

e
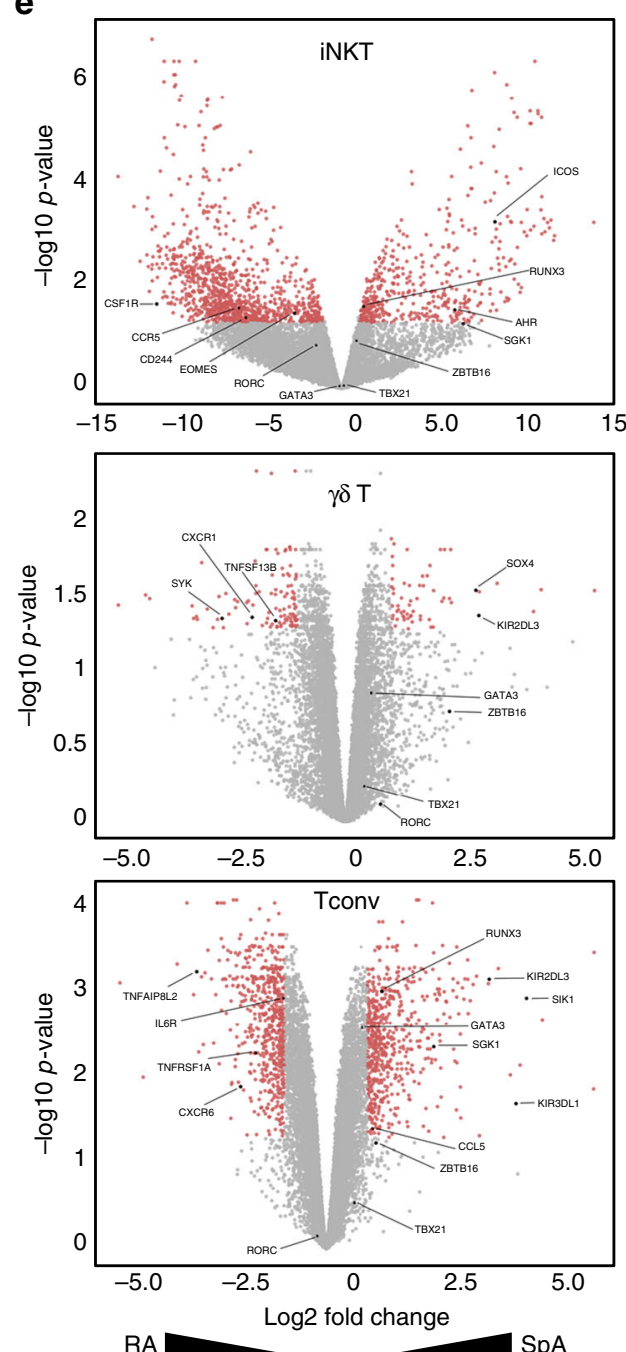

f

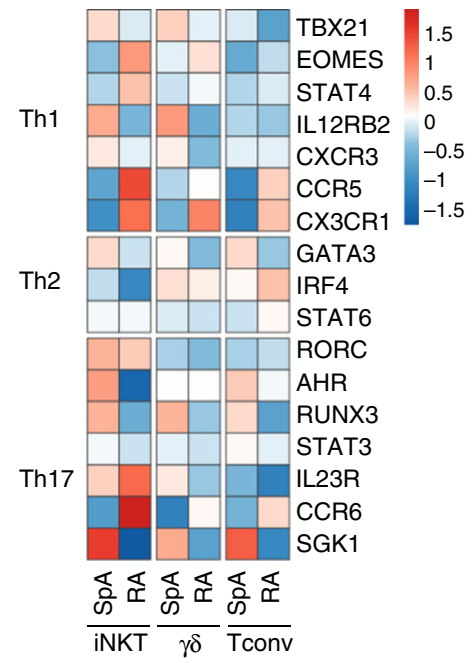

the investigated T cell subsets. Interestingly, for iNKT cells, there was clear upregulation of iNKT17 associated genes (AHR, SGK1, $R U N X 3$, and ICOS) in SpA and upregulation of iNKT1 associated genes (CD244, CSF1R, EOMES, CCR5) in RA. The overexpression $A H R$ and SGK1 mRNA in SpA vs. RA immune cells are of interest as they are known to be triggered by environmental signals. Indeed, AHR (Aryl Hydrocarbon Receptor) encodes for a ligand-activated nuclear transcription factor which has been shown to be expressed by iNKT17 cells, although rather promotes IL-22 secretion while suppressing IL-17 ${ }^{40}$. AHR activation is 
Fig. 5 iNKT and $\gamma \delta-T$ cells show different gene expression profiles in SpA and RA patients. a Frequencies of iNKT and $\gamma \delta-T$ cells in indicated groups of rheumatic patients (each dot represents data from a single patient). Statistical analyses $\left({ }^{\star} p<0.05,{ }^{\star \star} p<0.01\right)$ referred to comparisons of blood samples (patients vs. controls) and comparisons of SF as compared to paired blood samples of patients as done by ANOVA and paired $t$-tests, respectively (same for $\mathbf{b}$ and $\mathbf{c}$ ). (PB: black symbols; SF: red symbols) b Relative percentage of TCR $\gamma \delta$-hi cells and $\mathbf{c}$ IL-23R (lower panel) in $\gamma \delta$-T cells of samples taken from patients and controls. (PB: white bars; SF: red bars) d Principal component analysis (PCA) of transcriptomes (RNAseq data) of iNKT, $\gamma \delta-T$ and Tconv cells sorted from SpA $(n=7$, red) and RA patients $(n=5$, blue). Each sphere symbol represents an individual patient. e Differentially expressed genes are represented in Volcano plots after pairwise comparisons between indicated subsets of SpA and RA patients. Each symbol represents a single gene and genes of interest are highlighted (see also main text). Color coding refers to differentially expressed genes with adjusted p-value less than 0.05 and the log2FC less than -1 (higher expressed in RA derived cells) or greater than 1 (higher expressed in SpA). $\mathbf{f}$ Heatmap showing relative expression Th1, Th2, and Th17 related genes in indicated cell subsets from SpA and RA patients. Data throughout this figure are presented as mean \pm SEM

induced by naturally occurring compounds such as tryptophan metabolites (derived from microorganisms), next to synthetic polycyclic aromatic hydrocarbons and dioxin-like compounds ${ }^{47}$. SGK1 (Serum/Glucocorticoid Regulated Kinase 1) gene expression in iNKT cells has not been described yet, but may be critically important as this kinase is involved in salt induced signaling processes and it is able to promote Th17 function while suppressing Treg function ${ }^{48,49}$. In addition, $\gamma \delta$-T cells of SpA patients differed substantially from RA by a remarkable upregulation of SOX4, a high-mobility group (HMG) box transcription factor, key in $\gamma \delta 17$ cell-specific transcription program $^{50}$, whereas we noted that IL18RA (CXCR1) and BAFF were significantly higher expressed in RA. BAFF, is a key cytokine for $B$ cell activation and maturation, mainly expressed by myeloid cells and stromal cells, but it was recently also shown to be expressed by human TCR $\gamma \delta$ cells upon TGF- $\beta /$ IL-15 stimulation $^{51}$. Finally, Heatmap visualization of typical Th cell genes demonstrated that innate-like T cell subsets, especially iNKT, to be more skewed towards a Th17 cell associated profile in SpA as compared to RA (Fig. 5f)

In addition to these marked differences in innate-like $\mathrm{T}$ cells, also conventional $\mathrm{T}$ cells displayed some marked differences between SpA and RA. This included expression of several Killer cell immunoglobulin-like receptors (KIRs), transmembrane glycoproteins expressed by natural killer cells and subsets of $\mathrm{T}$ cells, which were upregulated in SpA patients. For example, KIR3DL1 was reported to act as a receptor for HLA-B27 homodimers $^{52}$ representing a mechanism by which HLA-B27 may be linked to aberrant $\mathrm{NK}$ and $\mathrm{T}$ cell responses in $\mathrm{SpA}^{53}$. By contrast, RA conventional T cells showed increased IL6R mRNA levels, as well as differences in expression of TNF signaling genes such as TNFRSF1A (encoding the TNFR1) and TNFAIP8L2 (TIPE2), both previously linked to RA disease pathogenesis ${ }^{54}$.

Collectively our data provides evidence for a "type 17 " skewed gene expression profile in SpA innate-like Tcells and this appears to be a discriminative feature compared to other forms of arthritis such as RA.

ROR $\gamma t$ inhibition selectively blocks innate-like $\mathrm{T}$ cells. Because Il-17 and/or Il-23 inhibition has demonstrated marked therapeutic efficacy in psoriasis, PsA and AS, a potential alternative strategy therefore includes ROR $\gamma \mathrm{t}$ inhibition by cost-effective small molecules. However, there are still several unanswered questions as to the impact of ROR $\gamma t$ blockade on IL-17 and/or IL23 mediated immunity as a consequence thereof and whether this impacts all Th17 related cytokines similarly. Several ROR $\gamma \mathrm{t}$ antagonists or inverse agonists (such as digoxin, SR1001 TMP778, GSK805, and MRL-871, JNJ-54271074) have been identified and tested in different disease models ${ }^{55-59}$. Here, we describe a new and very potent ROR $\gamma \mathrm{t}$ agonist/inhibitor, BIX119. This compound strongly bound to and selectively inhibited the human ROR $\gamma$ ligand-binding domain. In functional cell assays, BIX119 clearly abolished the induction of Th17 cells from human $\mathrm{CD} 4^{+}$ $\mathrm{T}$ cells and dose dependently suppressed IL-17 by reactivated Th17 cells (Fig. 6a), verifying that it is a selective and potent ROR $\gamma t$ inhibitor. It abolished IL-17 and IL-22 production in both $\mathrm{CD}^{+} \mathrm{T}$ cell and bulk PBMC cultures upon TCR stimulation (Fig. 6b and Supplementary Fig. 7A). Surprisingly, when BIX119 was added in cultures of IL-23 skewed human iNKT and $\gamma \delta$-T cells, we observed a significant inhibition of IL-17 but not of IL22 secretion (Fig. 6c-e). Likewise, restimulation of IL-23 skewed cells in the presence of BIX119 led to a selective blocking of IL-17 but not IL-22 secretion (Supplementary Fig. 7B).

Human iNKT cells display a profound heterogeneity with regard to IL-17 and IL-22 production. We found single IL-17 and single IL-22 producing cells, next to a significant subset of double (IL-17+IL-22+) cytokine producers (Supplementary Fig. 7C). Interestingly, IL-17+IL-22- and IL-17+IL-22+cells expressed equally high levels of ROR $\gamma t$ whereas single IL-17 producers clearly differed from other subsets by reduced T-bet levels (Fig. 6f). The IL-22 single producers had an intermediate level of ROR $\gamma$ t expression (Fig. 6f). We also subjected this dataset to FlowSOM analyses and found that BIX119 significantly inhibited induction of several IL-17 producing iNKT subsets (Fig. $6 \mathrm{~g}$ and Supplementary Fig. 8). ROR $\gamma$ t inhibition shifted the balance from IL-17 producing cells towards a iNKT1 phenotype, while also increasing the prevalence of particular subsets of IL-22 producing cells (Fig. 6g and Supplementary Fig. 8). Thus, ROR $\gamma t$ inhibition does not only seems to repress iNKT17 immunity, it bypasses subsets of IL23 induced IL-22 producing cells.

Our data show that innate-like T cells such as iNKT and $\gamma \delta$-T cells are expanded and functionally skewed towards Il-17 secretion in SpA, diseases in which barrier integrity loss in skin or gut seems to sensitize the disease. Importantly, these subsets characterized by a unique transcription factor profile dominated by ROR $\gamma \mathrm{t}$ are clearly expanded in the circulation and even more in inflamed joints, highlighting the importance of these subsets in SpA. Quite unexpectedly we found that innate-like T cells differ substantially from conventional Th17 cells in cytokine regulation by ROR $\gamma t^{57,60,61}$, Earlier in-vivo studies indicated that transient blocking of ROR $\gamma \mathrm{t}$ allows for selective targeting of Th17 cells without influencing protective ILC3 responses $^{62}$. In addition, pharmacological inhibition of ROR $\gamma \mathrm{t}$ can modulate TCRa gene rearrangement taken place in developing thymic $\mathrm{T}$ lymphocytes and by this moderately limited the $\mathrm{T}$ cell repertoire diversity in peripheral circulation ${ }^{63}$. It was not reported whether this influenced iNKT development in treated mice, a finding shown in patients with loss-of-function mutations in the RORC gene ${ }^{64}$. By unsupervised computational analysis of flow cytometry data we uncovered a previously unappreciated complexity of both human $\gamma \delta$-T and iNKT cells as we show that ROR $\gamma \mathrm{t}$ inhibition not only efficiently blocks Th17 function, but also ablated human IL-17 producing $\gamma \delta-T$ cells and iNKT cells while selectively sparing IL-22+ subsets. These results confirm that IL-22 can be secreted by innate-like $\mathrm{T}$ cells independently from RORC driven 
a
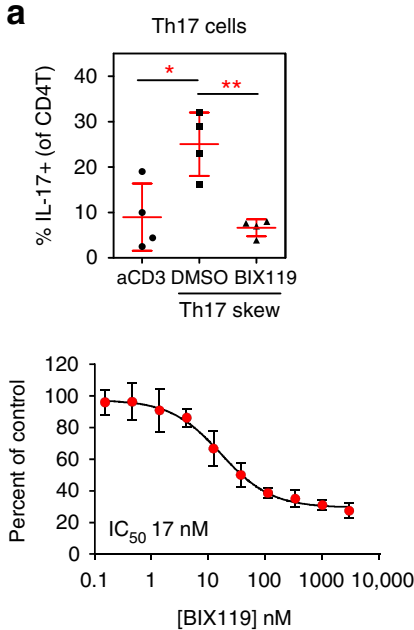

b
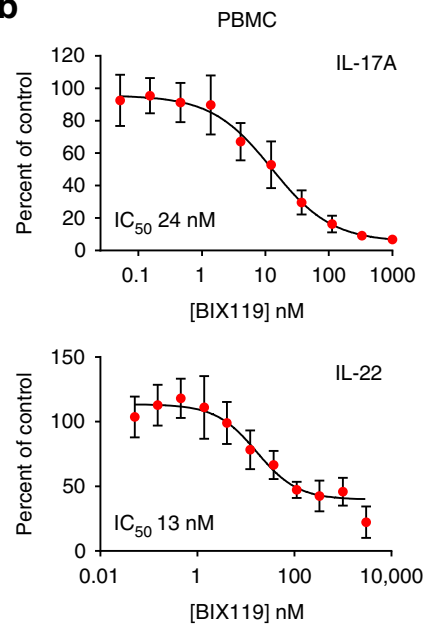

C

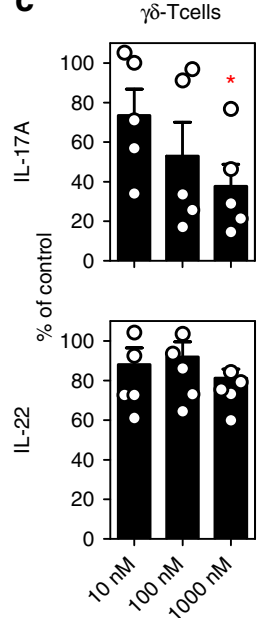

[BIX119]

f
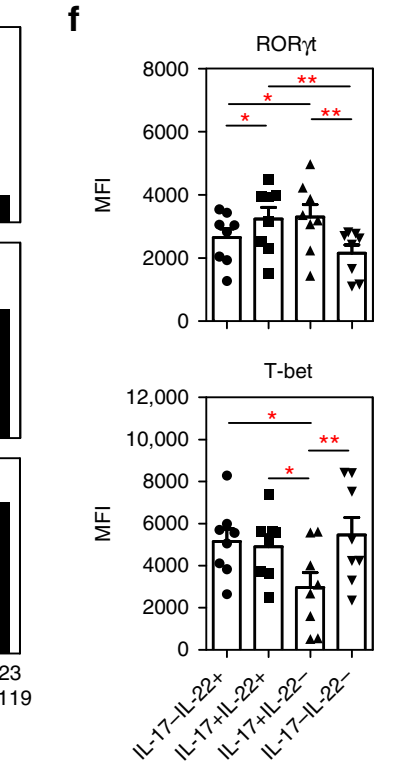

g

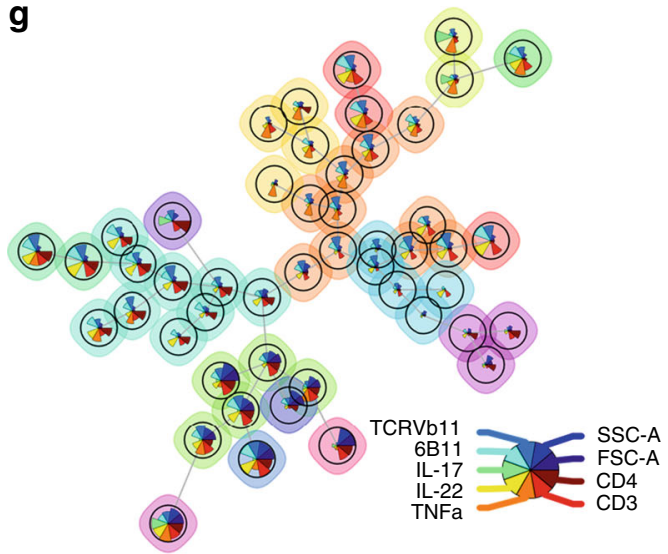

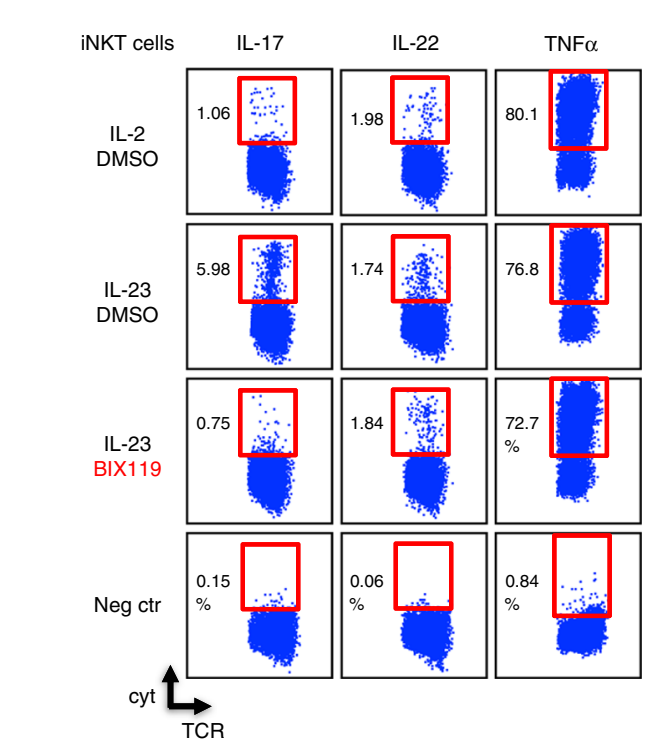

e
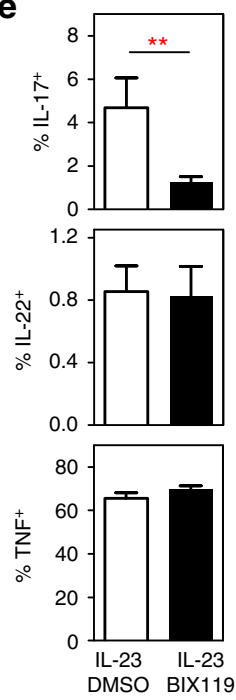

DMSO BIX119

d

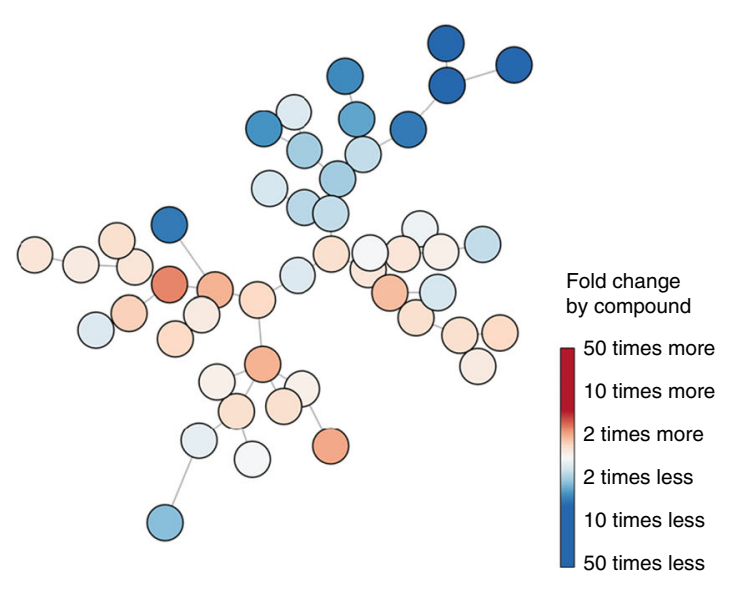

IL-17 production, potentially by AHR signaling. Given the established gut-joint axis in $\mathrm{SpA}$, this can be of clinical importance as IL-22 plays a protective role at gut barrier surfaces ${ }^{10}$, sites known to be enriched in innate-like $(\mathrm{T})$ cells. AHR signaling in gut mucosal ILC cells has been shown to be responsible for an IL-22 dependent modulation of immune

responses against mucosal microbiota and protecting mice from exacerbated colitis ${ }^{65,66}$. Given that IL-17 also mediated protective barrier function in the gut, these data point to a delicate and complex network of pro-inflammatory and anti-inflammatory signals involved in host protection. A shift in these immune processes in susceptible individuals may tip the balance toward 
Fig. 6 RORC inhibition selectively blocks innate-like T cell function. a Upper panel: healthy donor CD4+ T cells cultured under Th17 conditions for 7 days. Data is represented as \% IL-17+ cells as determined by intracellular flow cytometry $(n=4) .\left({ }^{\star} p<0.05,{ }^{\star \star} p<0.01\right.$ as determined by ANOVA). Lower panel: Skewed CD4+ Th17 cells were re-stimulated under Th17 conditions for $48 \mathrm{~h}$ and IL-17 levels were measured in the supernatants. Dose response of the ROR $\gamma t$ antagonist (BIX119) for three donors is shown. b Human PBMC activated with aCD3Ab and IL-23 for $72 \mathrm{~h}$. Dose response of the ROR $\gamma \mathrm{t}$ antagonist is shown for IL-17A and IL-22 secretion detected in culture supernatants by ELISA $(n=3)$. c $\gamma \delta$-T cells were activated with anti-CD3/CD28 beads and IL-23 cocktail for four days to produce IL-17A and IL-22 in the presence of increasing concentrations of ROR $\gamma$ t antagonist BIX119 $(n=5)$. ${ }^{*} p<0.05$ as compared to no compound (ANOVA) (d) PBMC were stimulated with $\alpha$ GalCer in the presence of IL-2 or IL-23 cocktail with addition of BIX119 (1 $\mu$ M) or DMSO. After 14 days, cells were restimulated with PMA/Cal (with BFA) for intracellular cytokine detection. One example representative for three independent experiments is shown. The negative control (neg ctr) consisted of IL-23 skewed cells cultured in BFA alone. e quantitative overview of frequency of IL-17, $\mathrm{IL}-22$ and TNF $\alpha+i N K T$ cells from assays described in $\mathrm{D}\left({ }^{\star \star} p<0.01\right.$ by paired $t$-tests). $\mathbf{f} \mathrm{IL}-23$ skewed iNKT cells were analyzed for ROR $\gamma \mathrm{t}$ and T-bet expression by intranuclear flow cytometry. Gating on cytokine producing iNKT cells was performed as illustrated in supplementary figure $7 \mathrm{C}\left({ }^{\star} p<0.05\right.$, ${ }^{\star \star} p<0.01$ ANOVA). $g$ FlowSOM visualization (as explained in Fig. 3F) of IL-23 skewed human iNKT cells $(n=3)$, cultured in the presence or absence of the BIX119 RORyt antagonist (compound). Pie chart shows markers used for automated clustering of iNKT cell types. Data throughout this figure are presented as mean \pm SEM

disease initiating events in $\mathrm{SpA}^{67}$. Whether AHR mediated responses are altered is appealing given the increased $A H R$ gene expression we observed in blood circulating iNKT cells in SpA patients. This however warrants additional investigation of innate-like $\mathrm{T}$ cells in gut samples from SpA patients ${ }^{67}$.

A decrease of blood iNKT cells and a relative enrichment of these cells in the joint compartment of SpA patients may also reflect shifts in iNKT cell subsets due to migration of pathogenic subsets toward sites of inflammation. In vivo evidence for such a scenario was very recently provided by the Kronenberg team showing an accumulation of iNKT cells in ankle joints upon progression of mannan induced arthritis in SKG mice ${ }^{68}$. Interestingly, this coincided with a progressive increase of iNKT17 cells in the inflamed joint tissue at the expense of protective IFN $\gamma$ secreting iNKT1 cells. Alternatively, iNKT1 cells in $\mathrm{SpA}$ could be more susceptible towards transition to an iNKT17 cell phenotype under inflammatory conditions ${ }^{40}$. T-bet acts as a repressor of the IL-23/IL-17 immune pathway ${ }^{69}$. So the low $\mathrm{T}$-bet expression in innate-like $\mathrm{T}$ cells observed in SpA patients could contribute itself to uncontrolled IL23 mediated pathology.

Collectively these data point to a distinctive regulation of innate-like T cells in SpA with a Th17-skewed phenotype and our data are clearly supportive for the progress in putting forward ROR $\gamma t$ antagonism, targeting these cells, as a potential promising new therapeutic approach.

\section{Methods \\ Human samples. Blood samples were obtained from 27 healthy control subjects and 33 patients with newly diagnosed SpA. Synovial fluid (SF) was obtained from patients with an active knee synovitis and an indication for aspiration. Rheumatoid arthritis (RA, $n=17)$ and Crystal-induced arthropathies (CrA, $n=11)$ patients, were included as a chronic and acute inflammatory arthritis comparison group, respectively. For details on the demographic and clinical characteristics of the patients, see Supplementary Table 1. Diagnosis and classification of patients was made by staff rheumatologists following the EULAR/ American College of Rheu- matology (ACR) 2010 or ASessment in Ankylosing Spondylitis (ASAS) 2010 cri- teria. Patients were treatment naïve or under treatment with a NSAID and/or DMARD (Methotrexate, Sulfasalazin), but naïve for treatment with biologicals at the time op sampling (see Supplementary Table 1). Ultrasound guided synovial biopsy sampling of knee joints was done by an experienced rheumatologist in our early arthritis clinic. At least six biopsies were taken to ensure enough cellular material ( $>500,000$ cells) could be extracted for flow analyses. This study was approved by the local ethics committee of Ghent University Hospital and all patients and healthy controls gave written informed consent.}

Cell isolation. PBMC and SFMC were directly isolated from PB and SF samples as described before ${ }^{70}$. Synovial tissue samples were digested with a mixture of Collagenase IV and VIII (Sigma, both $1 \mathrm{mg} / \mathrm{ml}$ ) for $30 \mathrm{~min}$ at $30^{\circ} \mathrm{C}$ under continuous stirring conditions. Digested samples were filtrated $(40 \mu \mathrm{m})$, washed and prepared for flow cytometric analyses as indicated below. Blood $\gamma \delta$-T cells $(\mathrm{TCR} \gamma \delta+)$ and iNKT cells (TCRVa24+ TCRV $\beta 11+$ ) were sorted on a FACSAria III (BD). The latter cells were expanded by $6 \mathrm{~B} 11$ stimulation as described in ref. ${ }^{71}$. IL-17+
iNKT cells were isolated by means of an adapted IL-17 Capture Assay (Cell Enrichment and Detection Kit, Miltenyi Biotec). Briefly, two weeks after aGalCer (100 ng/ml) and IL-23 stimulation, PBMC were restimulated with Cytostim (Miltenyi Biotec; crosslinking TCR and MHC) for $4 \mathrm{~h}$ at $37^{\circ} \mathrm{C}$. Subsequently, cells were labeled with IL-17 Catch Reagent, by which cell secreted IL-17 is bound at the cell membrane. Finally, cells are labeled with an APC labeled IL-17 detection antibody, next to 7-aminoactinomycin $\mathrm{D}$ (7-AAD; for life/dead discrimination) and antibodies directed against TCRVa24 and TCRV $\beta 11$, prior to FACSorting of IL-17+ TCRva24TCRv $\beta 11^{+} 7-\mathrm{AAD}^{\text {neg }}$ cells. A negative (no Cytostim) and positive (PMA/CaI/BFA) control condition was used in each experiment.

Cell cultures. For iNKT assays, PBMC were cultured in 24 -well plates $\left(1.5 \times 10^{6}\right.$ cells/well) for 14days in the presence of aGalCer $(100 \mathrm{ng} / \mathrm{mL}$, in house made) and IL-23 (20 ng/mL), IL-1 $\beta$ (10 ng/mL), TGF $\beta 1$ ( $5 \mathrm{ng} / \mathrm{mL}$; all eBioscience), and IL-2 ( $5 \mathrm{U} / \mathrm{mL}$, Roche) or IL-2 alone. Similar experiments were performed with sorted iNKT and $\gamma \delta$-T cells in U bottom 96 well plates (50,000 cells/well) where we added 50,000 irradiated (40 Gy) T cell depleted PBMC cells (using CD2 dynabeads, ThermoFisher) as feeder/antigen presenting cells. Cytokine titers in supernatants were then determined by ELISA (ebioscience) or multiplex protein assays (MSD).

SFMC depletion assay. SFMC from SpA patients $(n=3)$ were split in two equal fractions (I and II). Both fractions underwent a similar sorting procedure to exclude a potential bias on functionality. Fraction I was labeled only with 7-AAD to exclude dead cells. Cells from fraction II were labeled with anti- human TCRV $\beta 11$, $6 \mathrm{~B} 11$ and TCR $\gamma \delta$ and $7-\mathrm{AAD}$. Fraction I and II were acquired on a FACSAria III sorter $(\mathrm{BD})$ at the same flow rate and gated for live lymphocytes (7-AADneg FSClo/ SSC $\left.^{\text {lo }}\right)$. Fraction II was depleted of iNKT (TCRV $\left.\beta 11+6 \mathrm{~B} 11+\right)$ and $\gamma \delta$-T cells $(\mathrm{TCR} \gamma \delta+)$ cells by negative gating (see Fig. 4c). Equal cell numbers (min. 300,000) from sorted SFMC and SFMC cells depleted of iNKT and TCR $\gamma \delta$ cells $(\triangle$ SFMC) were cultured in 96 flat bottom plates. For TCR stimulation, wells were (prior to cell adition) plate coated with aCD3Ab and aCD28Ab (both at $5 \mu \mathrm{g} / \mathrm{ml}$ in PBS, BD) overnight at $4{ }^{\circ} \mathrm{C}$. Wells indicated for non-TCR stimulated conditions were incubated with PBS only. Wells were washed extensively before cells were added. IL-23 $(20 \mathrm{ng} / \mathrm{ml})$ together with IL-1 $\beta(10 \mathrm{ng} / \mathrm{ml})$ and TGF $\beta 1(5 \mathrm{ng} / \mathrm{ml})$ resuspended in culture medium was added to indicated wells ("IL-23" condition). Non cytokine stimulated wells were incubated with medium only ("no IL-23"). Supernatants of cultures were collected at $72 \mathrm{~h}$ of culture and in levels of IL-17, IL-22, TNFa, and IFN $\gamma$ were determined by ELISA (eBiosciences).

Flow cytometry. Eight-ten color flow cytometry was applied to PBMC and SFMC samples and isolated (cultured) cells as described before ${ }^{70,71}$, using fluorochrome conjugated antibodies as summarized in Supplementary Table 2. iNKT cells $(\mathrm{CD} 3+6 \mathrm{~B} 11+\mathrm{TCRV} \beta 11+), \gamma \delta$-T cells $(\mathrm{CD} 3+\mathrm{TCR} \gamma \delta+)$ and CD161+ and CD161 - conventional Tconv ( $\mathrm{T}$ cells excluded from innate-like T cells, CD3+ TCR $\gamma \delta-$ TCRVa7.2-TCRVa24Ja18-) were gated as shown in Supplementary Fig. 1A. Human aGalCer loaded CD1d tetramer (in house produced) was used to verify iNKT stainings as indicated in Supplementary Figure 1E-F). FcR Blocking Reagent (Miltenyi Biotec) was used to exclude aspecific FcR binding of Abs. For flowcytometric analyses of cultured cells, Ab staining was preceded by incubation with viable cell discriminating reagents (LIVE/DEAD ${ }^{\circledR}$ Fixable Violet Dead Cell Stain Kit, ThermoFisher or Fixable Viability Stain 520, BD). A minimum of 500 iNKT and $3000 \gamma \delta$-T cells were acquired on a FACS Canto II or Aria III machine (BD) to guarantee accurate calculations of the expression of differentiation markers/transcription factors by these cells. Data analyses and graph presentations were done by means of FlowJo software, v9.8 and 10.2 (Treestar). Prior to intracellular staining of transcription factors, the cells were fixed and permeabilized using the reagents and following the instructions of the FoxP3 intracellular staining kit (eBioscience). For cytokine stainings, cells were collected and restimulated for $4 \mathrm{~h}$ with PMA (25 ng/ $\mathrm{mL})$, calcium-ionomycin $(1 \mu \mathrm{g} / \mathrm{mL}$; both from Sigma $)$ and brefeldin $\mathrm{A}(10 \mathrm{ng} / \mathrm{mL}$; 
BD). Subsequently, cells were processed using the Cytofix/Cytoperm kit (BD) as described before ${ }^{70}$

$R O R C$ and $I l 23 R$ mRNA expression on specific $\mathrm{T}$ cell subsets was measured by means of a PrimeFlow RNA Assay (eBioscience), using VA1 and VA4 probes, respectively, and following the manufacturer's instructions. Proper positive and negative controls (RPL13A and FMO) were included in each assay.

qPCR. Cells were lysed using RLT lysis buffer supplemented with $\beta$ mercaptoethanol (Qiagen) and were kept at $-80^{\circ} \mathrm{C}$ until analysis. RNA extraction and cDNA preparation were conducted using the RNeasy mini kit (Qiagen) and the QuantiTect Reverse Transcription Kit (Qiagen), according to the manufacturer's instructions. qPCR reactions were performed in duplicate using a LightCycler 480 system (Roche). QuantiTect primer assays for IL17A, IL17F, IL22, RORC, and IL23R genes were ordered from Qiagen. Reference (housekeeping) genes were designed, validated and tested for stable expression (including Gapdh, $\beta$-actin and HPRT) and data were analyzed using qbase+ (Biogazelle).

RNASeq. PBMC were isolated from freshly taken blood samples of treatment naïve SpA patients $(n=7)$ and RA patients $(n=5)$ as described above. Cells were labeled with anti-human TCRV $\beta 11,6 \mathrm{~B} 11, \mathrm{CD} 161, \mathrm{CD} 3, \mathrm{TCR} \gamma \delta$ and 7-AAD to exclude dead cells. iNKT $(\mathrm{CD} 3+\mathrm{TCRVb} 11+6 \mathrm{~B} 11+), \gamma \delta$-T cells $(\mathrm{CD} 3+\mathrm{TCR} \gamma \delta+)$ cells and Tconv (CD3 + CD161-; negative for iNKT and $\gamma \delta$-T markers) were sorted on a FACSAria III (BD) and immediately stored in RLT (Qiagen) $+1 \% \beta$ Mercaptoethanol (Sigma) at $-80^{\circ} \mathrm{C}$. RNA was isolated by means of the RNeasy Micro kit following manufacturer's instructions (Qiagen). RNA concentration and purity were determined spectrophotometrically using the Nanodrop ND-1000 (Nanodrop Technologies) and RNA integrity was assessed using a Bioanalyser 2100 (Agilent). Per sample, an amount of $100 \mathrm{pg}$ (iNKT) or $1 \mathrm{ng}$ ( $\gamma \delta$ and Tconv) of total RNA was used as input for the SMART-Seq v4 Ultra Low Input RNA protocol (version 091817) from Takara Bio USA, Inc. Subsequently, $1 \mathrm{ng}$ (iNKT) or $2 \mathrm{ng}$ ( $\gamma \delta$-T and Tconv samples) of purified cDNA was sheared to $300 \mathrm{bp}$ using the Covaris M220. From the sheared material, sequencing libraries were prepared with the NEBNext Ultra DNA Library Prep Kit for Illumina (version 6.0-2/18), according to the manufacturer's protocol including a size selection to $250 \mathrm{bp}$ insert size. Sequence-libraries of each sample were finally equimolarly pooled and sequenced on 4 NextSeq500 v2 flow-cell at $1 \times 75$ bp $(76-8-8-0)$. The preprocessing of the RNA sequencing data was done by Trimmomatic. The adapters were cut off, and reads were trimmed when the quality dropped below 20 . Reads with a length $<35$ were discarded. All samples passed quality control based on the results of FastQC. Reads were mapped to the human reference genome (hg19) via Tophat2 and counted via HTSeqCount. Samples were subsequently analyzed using R/Bioconductor, and the $\mathrm{R}$ package limma was used to normalize the data and to do differential expression analysis. The 3 cell types were analyzed separately and we corrected for batch effects using ComBat of the R package sva. For the analysis of differentially expressed (DE) genes, we applied a stringency level where the adjusted p-value was less than 0.05 and the $\log 2 \mathrm{FC}$ was less than -1 or greater than 1. For the heatmap we scaled the log2 expression values per gene by calculating the mean expression per gene and then substracting that mean value of each expression value.

ROR $\gamma \mathbf{t}$ compound. The ROR $\gamma t$ agonist/inhibitor, BIX119, was discovered through screening a small-molecule compound library. BIX119 strongly bound to the human ROR $\gamma$ ligand-binding domain (LBD) and was active in a ROR $\gamma$ LBD reporter assay (Kd for ROR $\gamma \mathrm{LBD}-65 \mathrm{nM}$; IC50 for ROR $\gamma \mathrm{LBD}$ reporter assay $260 \mathrm{nM}$ ). The compound showed high selectivity towards ROR $\gamma \mathrm{t}$ as demonstrated by a lack of significant activity against ROR $\alpha$ (IC50 $>10 \mu \mathrm{M}$ ) and ROR $\beta$ (IC50 > than $6 \mu \mathrm{M}$ ). In addition, BIX119 was inactive against a panel of 25 nuclear receptors up to $10 \mu \mathrm{M}$. BIX119 potently inhibited IL-17A secretion from human PBMCs activated with anti-CD3Abs and IL-23, as well as CD4 ${ }^{+}$Th17 cells stimulated with anti-CD3/CD28/CD2 beads and a Th17 cytokine cocktail including IL-1 $\beta$, IL-23, IL-6, and TGF $\beta$ (IC $5010 \mathrm{nM}$ and $17 \mathrm{nM}$, respectively). Levels of IL-17 and IL-22 in supernatants of compound experiments were determined by means of ELISA using respecitively the human IL-17A Tissue Culture Kit (Meso Scale Discovery) and the Human IL-22 Quantikine Kit (R\&D Systems).

FlowSOM. Multiparameter flow cytometry data derived from patient and control samples, were subjected to FlowSOM ${ }^{21}$, which uses a self-organizing map (SOM) to cluster and visualize the selected cell subsets (iNKT, TCR $\gamma \delta$, and Tconv) in different nodes based on the expression of distinct markers included in the flow $\mathrm{Ab}$ panel. For each subset, an aggregated file was created containing the cells from the different samples, compensated and transformed as specified by the FlowJo workspace and for the resulting aggregated files, a FlowSOM tree was trained. A grid of 7 by 7 nodes, resulting in 49 clusters was used, which are visualized using the Minimal Spanning Tree visualization. Scaling was set to FALSE and the number of metaclusters to 10 , all other parameters were kept as default. The legends in the figures indicate which markers were used for clustering. The individual files were mapped onto the trees to compute the percentage of cells assigned to each cluster. The mean percentage per sample group was determined, and the $\log 10$ fold change was computed to visualize the differences between the groups.
The $\log 10$ fold change was manually set to -2 (or a decrease by a 100 times less) if no cells where present in the patient group (corresponding with a theoretical fold change of -infinity). A similar strategy was applied for the compound data, with $\log 10$ fold change calculated for the condition with compound vs. no compound (only vehicle). Additional information related to the interpretation of the FlowSOM tree figures is included in the figure legends.

Statistics. Data were analyzed using Prism software v5.0 (GraphPad) and SPSS version 23 software. The Shapiro-Wilk test was used to test normality of the data. Statistic tests (and annotations) are mentioned in the figures and figure legends. An overview of statistics for Fig. If is provided in the Supplementary Table 3. In general, the two-sided non-parametric Mann-Whitney $U$ test was performed for comparison of non-normality numeric data, whereas two-sided student's (paired) $t$ test were used for normally distributed data. One-way analysis of variance (ANOVA) were used for multi-group analyses of normally distributed data. Data is presented as mean \pm SEM unless stated otherwise. All the statistical assessments were made at the $95 \%$ confidence interval; $p<0.05$ was considered to be statistically significant.

Reporting Summary. Further information on experimental design is available in the Nature Research Reporting Summary linked to this article.

\section{Data availability}

RNA-seq data from this study is deposited in GEO with accession code GSE122624. Other data that support the findings of this study are available from the corresponding authors upon reasonable request.

Received: 24 July 2017 Accepted: 4 December 2018

Published online: 02 January 2019

\section{References}

1. Rudwaleit, M. et al. The Assessment of Spondylo Arthritis International Society classification criteria for peripheral spondyloarthritis and for spondyloarthritis in general. Ann. Rheum. Dis. 70, 25-31 (2011).

2. Jacques, P. \& Elewaut, D. Joint expedition: linking gut inflammation to arthritis. Mucosal Immunol. 1, 364-371 (2008).

3. Venken, K. \& Elewaut, D. IL-23 responsive innate-like T cells in spondyloarthritis: the less frequent they are, the more vital they appear. Curr. Rheumatol. Rep. 17, 30 (2015).

4. Baeten, D. et al. Secukinumab, an interleukin-17A inhibitor, in ankylosing spondylitis. N. Engl. J. Med. 373, 2534-2548 (2015).

5. Baeten, D. et al. Risankizumab, an IL-23 inhibitor, for ankylosing spondylitis results of a randomised, double-blind, placebo-controlled, proof-of-concept, dose-finding phase 2 study. Ann. Rheumatic Dis. 77, 1295-1302 (2018).

6. Targan, S. R. et al. A randomized, double-blind, placebo-controlled study to evaluate the safety, tolerability, and efficacy of AMG 827 in subjects with moderate to severe Crohn's disease. Gastroenterology 143, E26-E26 (2012).

7. Blanco, F. J. et al. Secukinumab in active rheumatoid arthritis: a phase iii randomized, double-blind, active comparator- and placebo-controlled study. Arthritis Rheumatol. 69, 1144-1153 (2017).

8. Maxwell, J. R. et al. Differential roles for interleukin-23 and interleukin-17 in intestinal immunoregulation. Immunity 43, 739-750 (2015).

9. Lee, J. S. et al. Interleukin-23-independent IL-17 production regulates intestinal epithelial permeability. Immunity 43, 727-738 (2015).

10. Sonnenberg, G. F., Fouser, L. A. \& Artis, D. Border patrol: regulation of immunity, inflammation and tissue homeostasis at barrier surfaces by IL-22. Nat. Immunol. 12, 383-390 (2011).

11. Ivanov, I. I. et al. The orphan nuclear receptor RORgammat directs the differentiation program of proinflammatory IL-17+ T helper cells. Cell 126, $1121-1133$ (2006).

12. Zhong, C. \& Zhu, J. Small-molecule RORgammat antagonists: one stone kills two birds. Trends Immunol. 38, 229-231 (2017).

13. Gaffen, S. L., Jain, R., Garg, A. V. \& Cua, D. J. The IL-23-IL-17 immune axis: from mechanisms to therapeutic testing. Nat. Rev. Immunol. 14, 585-600 (2014).

14. Sherlock, J. P. et al. IL-23 induces spondyloarthropathy by acting on RORgammat + CD3+CD4-CD8- entheseal resident T cells. Nat. Med. 18, 1069-1076 (2012).

15. Venken, K. \& Elewaut, D. New immune cells in spondyloarthritis: key players or innocent bystanders? Best. Pract. Res. Clin. Rheumatol. 29, 706-714 (2015).

16. Rossjohn, J., Pellicci, D. G., Patel, O., Gapin, L. \& Godfrey, D. I. Recognition of CD1d-restricted antigens by natural killer T cells. Nat. Rev. Immunol. 12, 845-857 (2012). 
17. Lee, Y. J., Holzapfel, K. L., Zhu, J., Jameson, S. C. \& Hogquist, K. A. Steadystate production of IL-4 modulates immunity in mouse strains and is determined by lineage diversity of iNKT cells. Nat. Immunol. 14, 1146-1154 (2013).

18. Chien, Y. H., Meyer, C. \& Bonneville, M. gammadelta T cells: first line of defense and beyond. Annu. Rev. Immunol. 32, 121-155 (2014).

19. Martin, B., Hirota, K., Cua, D. J., Stockinger, B. \& Veldhoen, M. Interleukin17-producing gammadelta $\mathrm{T}$ cells selectively expand in response to pathogen products and environmental signals. Immunity 31, 321-330 (2009).

20. Caccamo, N. et al. Differentiation, phenotype, and function of interleukin-17producing human Vgamma9Vdelta2 T cells. Blood 118, 129-138 (2011).

21. Van Gassen, S. et al. FlowSOM: Using self-organizing maps for visualization and interpretation of cytometry data. Cytom. A. 87, 636-6 45 (2015).

22. Maggi, L. et al. CD161 is a marker of all human IL-17-producing T-cell subsets and is induced by RORC. Eur. J. Immunol. 40, 2174-2181 (2010).

23. Haas, J. D. et al. Development of interleukin-17-producing gammadelta $\mathrm{T}$ cells is restricted to a functional embryonic wave. Immunity 37, 48-59 (2012)

24. Prinz, I., Silva-Santos, B. \& Pennington, D. J. Functional development of gammadelta T cells. Eur. J. Immunol. 43, 1988-1994 (2013).

25. Paget, C. et al. CD3bright signals on gammadelta T cells identify IL-17Aproducing Vgamma6Vdelta1+T cells. Immunol. Cell Biol. 93, 198-212 (2015).

26. Savage, A. K. et al. The transcription factor PLZF directs the effector program of the NKT cell lineage. Immunity 29, 391-403 (2008).

27. Kovalovsky, D. et al. The BTB-zinc finger transcriptional regulator PLZF controls the development of invariant natural killer $\mathrm{T}$ cell effector functions. Nat. Immunol. 9, 1055-1064 (2008).

28. Rahimpour, A. et al. Identification of phenotypically and functionally heterogeneous mouse mucosal-associated invariant T cells using MR1 tetramers. J. Exp. Med. 212, 1095-1108 (2015).

29. Kreslavsky, T. et al. TCR-inducible PLZF transcription factor required for innate phenotype of a subset of gammadelta T cells with restricted TCR diversity. Proc. Natl Acad. Sci. USA 106, 12453-12458 (2009).

30. Constantinides, M. G., McDonald, B. D., Verhoef, P. A. \& Bendelac, A. A committed precursor to innate lymphoid cells. Nature 508, 397-401 (2014)

31. Eidson, M. et al. Altered development of NKT cells, gammadelta T cells, CD8 T cells and NK cells in a PLZF deficient patient. PLoS One 6, e24441 (2011).

32. Singh, S. P. et al. PLZF regulates CCR6 and is critical for the acquisition and maintenance of the Th17 phenotype in human cells. J. Immunol. 194, 4350-4361 (2015).

33. Cohen, N. R. et al. Shared and distinct transcriptional programs underlie the hybrid nature of iNKT cells. Nat. Immunol. 14, 90-99 (2013).

34. Lee, Y. J. et al. Lineage-specific effector signatures of invariant NKT cells are shared amongst gammadelta T, innate lymphoid, and Th cells. J. Immunol. 197, 1460-1470 (2016).

35. Lynch, L. et al. Regulatory iNKT cells lack expression of the transcription factor PLZF and control the homeostasis of T(reg) cells and macrophages in adipose tissue. Nat. Immunol. 16, 85-95 (2015).

36. Lee, Y. J. et al. Tissue-specific distribution of iNKT cells impacts their cytokine response. Immunity 43, 566-578 (2015).

37. Thomas, S. Y. et al. PLZF induces an intravascular surveillance program mediated by long-lived LFA-1-ICAM-1 interactions. J. Exp. Med. 208, 1179-1188 (2011).

38. Lu, Y., Cao, X., Zhang, X. \& Kovalovsky, D. PLZF controls the development of fetal-derived IL-17+ Vgamma6+ gammadelta T cells. J. Immunol. 195 4273-4281 (2015).

39. Awasthi, A. et al. Cutting edge: IL-23 receptor gfp reporter mice reveal distinct populations of IL-17-producing cells. J. Immunol. 182, 5904-5908 (2009)

40. Moreira-Teixeira, L. et al. Proinflammatory environment dictates the IL-17producing capacity of human invariant NKT cells. J. Immunol. 186, 5758-5765 (2011).

41. Guilliams, M. et al. Unsupervised high-dimensional analysis aligns dendritic cells across tissues and species. Immunity 45, 669-684 (2016).

42. Gracey, E. et al. IL-7 primes IL-17 in mucosal-associated invariant T (MAIT) cells, which contribute to the Th17-axis in ankylosing spondylitis. Ann. Rheum. Dis. 75, 2124-2132 (2016)

43. Ciccia, F. et al. Type 3 innate lymphoid cells producing IL-17 and IL-22 are expanded in the gut, in the peripheral blood, synovial fluid and bone marrow of patients with ankylosing spondylitis. Ann. Rheum. Dis. 74, 1739-1747 (2015).

44. Al-Mossawi, M. H. et al. Unique transcriptome signatures and GM-CSF expression in lymphocytes from patients with spondyloarthritis. Nat. Commun. 8, 1510 (2017).

45. Kugyelka, R. et al. Enigma of IL-17 and Th17 cells in rheumatoid arthritis and in autoimmune animal models of arthritis. Mediat. Inflamm. 2016, 6145810 (2016).

46. So, A. K. \& Martinon, F. Inflammation in gout: mechanisms and therapeutic targets. Nat. Rev. Rheumatol. 13, 639-647 (2017).
47. Denison, M. S. \& Nagy, S. R. Activation of the aryl hydrocarbon receptor by structurally diverse exogenous and endogenous chemicals. Annu. Rev. Pharmacol. Toxicol. 43, 309-334 (2003).

48. Kleinewietfeld, M. et al. Sodium chloride drives autoimmune disease by the induction of pathogenic TH17 cells. Nature 496, 518-522 (2013).

49. Wu, C. et al. Induction of pathogenic TH17 cells by inducible salt-sensing kinase SGK1. Nature 496, 513-517 (2013).

50. Malhotra, N. et al. A network of high-mobility group box transcription factors programs innate interleukin-17 production. Immunity 38, 681-693 (2013).

51. Peters, C., Hasler, R., Wesch, D. \& Kabelitz, D. Human Vdelta2 T cells are a major source of interleukin-9. Proc. Natl Acad. Sci. USA 113, 12520-12525 (2016).

52. Kollnberger, $\mathrm{S}$. et al. Cell-surface expression and immune receptor recognition of HLA-B27 homodimers. Arthritis Rheum. 46, 2972-2982 (2002).

53. Bowness, P. Hla-B27. Annu. Rev. Immunol. 33, 29-48 (2015).

54. Schett, G., Elewaut, D., McInnes, I. B., Dayer, J. M. \& Neurath, M. F. How cytokine networks fuel inflammation: toward a cytokine-based disease taxonomy. Nat. Med. 19, 822-824 (2013).

55. Huh, J. R. et al. Digoxin and its derivatives suppress TH17 cell differentiation by antagonizing RORgammat activity. Nature 472, 486-490 (2011).

56. Solt, L. A. et al. Suppression of TH17 differentiation and autoimmunity by a synthetic ROR ligand. Nature 472, 491-494 (2011).

57. Xiao, S. et al. Small-molecule RORgammat antagonists inhibit $\mathrm{T}$ helper 17 cell transcriptional network by divergent mechanisms. Immunity 40, 477-489 (2014).

58. Scheepstra, M. et al. Identification of an allosteric binding site for RORgammat inhibition. Nat. Commun. 6, 8833 (2015).

59. Xue, X. et al. Pharmacologic modulation of RORgammat translates to efficacy in preclinical and translational models of psoriasis and inflammatory arthritis. Sci. Rep. 6, 37977 (2016).

60. de Wit, J. et al. RORgammat inhibitors suppress $\mathrm{T}(\mathrm{H}) 17$ responses in inflammatory arthritis and inflammatory bowel disease. J. Allergy Clin. Immunol. 137, 960-963 (2016).

61. Chang, M. R., Lyda, B., Kamenecka, T. M. \& Griffin, P. R. Pharmacologic repression of retinoic acid receptor-related orphan nuclear receptor gamma is therapeutic in the collagen-induced arthritis experimental model. Arthritis Rheumatol. 66, 579-588 (2014)

62. Withers, D. R. et al. Transient inhibition of ROR-gammat therapeutically limits intestinal inflammation by reducing TH17 cells and preserving group 3 innate lymphoid cells. Nat. Med. 22, 319-323 (2016).

63. Guo, Y. et al. Inhibition of ROR gamma T Skews TCR alpha gene rearrangement and limits T cell repertoire diversity. Cell Rep. 17, 3206-3218 (2016).

64. Okada, S. et al. IMMUNODEFICIENCIES. Impairment of immunity to Candida and Mycobacterium in humans with bi-allelic RORC mutations. Science 349, 606-613 (2015).

65. Zelante, T. et al. Tryptophan catabolites from microbiota engage aryl hydrocarbon receptor and balance mucosal reactivity via interleukin-22. Immunity 39, 372-385 (2013).

66. Qiu, J. et al. Group 3 innate lymphoid cells inhibit T-cell-mediated intestinal inflammation through aryl hydrocarbon receptor signaling and regulation of microflora. Immunity 39, 386-399 (2013).

67. Mortier, C., Govindarajan, S., Venken, K. \& Elewaut, D. It takes “Guts” to cause joint inflammation: role of innate-like T cells. Front. Immunol. 9, 1489 (2018).

68. Zhao, M. et al. Altered thymic differentiation and modulation of arthritis by invariant NKT cells expressing mutant ZAP70. Nat. Commun. 9, 2627 (2018).

69. Lazarevic, V. et al. T-bet represses $\mathrm{T}(\mathrm{H}) 17$ differentiation by preventing Runx1-mediated activation of the gene encoding RORgammat. Nat. Immunol. 12, 96-104 (2011)

70. Melis, L., Van, P. L., Pircher, H., Venken, K. \& Elewaut, D. Senescence marker killer cell lectin-like receptor G1 (KLRG1) contributes to TNF-alpha production by interaction with its soluble E-cadherin ligand in chronically inflamed joints. Ann. Rheum. Dis. 73, 1223-1231 (2014).

71. Venken, K. et al. Bacterial CD1d-restricted glycolipids induce IL-10 production by human regulatory $\mathrm{T}$ cells upon cross-talk with invariant NKT cells. J. Immunol. 191, 2174-2183 (2013).

\section{Acknowledgements}

We would like to thank Dr. Lode Melis, Dr. Els Louagie, Mrs. Magalie Vanhouteghem, Mrs. Valerie Redant and Mr. Leslie Naesens for technical assistance and Dr. Liesbet van Praet and Dr. Philippe Carron (all UZGent) for collecting patient samples and characteristics. We acknowledge Profs. David Vermijlen (Université libre de Bruxelles) and Tom Taghon (UGent) for their kind gift of $\delta 3 \mathrm{Ab}$. Koen Venken was supported by FWO$\mathrm{Vl}$ (Fonds voor Wetenschappelijk Onderzoek Vlaanderen). D.E. is supported by FWOVl, Research Council of Ghent University and interuniversity Attraction Pole grant Devrepair from Belspo Agency (project P7/07) and a FWO Excellence of Science (EOS) Grant. M.E.L., K.H., A.L.W., R.H., M.T., D.S., C.H., J.W., C.W., and G.N. are employees of Boehringer Ingelheim (privately held company). 


\section{Author contributions}

K.V.: Designed and performed experiments, analyzed data and wrote the manuscript. C. M., T.D., J.C., E.V., and N.S. performed experiments. K.H., A.L.W., R.H., M.T., D.S., C. H., J.W., M.E.L., C.T.W., and G.N. designed, performed experiments and analyzed data with regard to the ROR $\gamma$ t compound. P.J., G.V., H.C., Y.P., L.G., R.W., F.V.D.B. contributed with patient samples. S.V.G. helped with FlowSOM analyses. L.M. and Y.S. performed the bioinformatics analyses (RNAseq data). S.V.C. provided reagents. D.E. supervised the work, designed the study, and edited the manuscript.

\section{Additional information}

Supplementary Information accompanies this paper at https://doi.org/10.1038/s41467018-07911-6.

Competing interests: The authors declare no competing interests.

Reprints and permission information is available online at http://npg.nature.com/ reprintsandpermissions/
Publisher's note: Springer Nature remains neutral with regard to jurisdictional claims in published maps and institutional affiliations.

(c) (i) Open Access This article is licensed under a Creative Commons Attribution 4.0 International License, which permits use, sharing, adaptation, distribution and reproduction in any medium or format, as long as you give appropriate credit to the original author(s) and the source, provide a link to the Creative Commons license, and indicate if changes were made. The images or other third party material in this article are included in the article's Creative Commons license, unless indicated otherwise in a credit line to the material. If material is not included in the article's Creative Commons license and your intended use is not permitted by statutory regulation or exceeds the permitted use, you will need to obtain permission directly from the copyright holder. To view a copy of this license, visit http://creativecommons.org/licenses/by/4.0/.

(C) The Author(s) 2019 